\title{
Starobinsky Inflation: From Non-SUSY to SUGRA Realizations
}

\author{
Constantinos Pallis and Nicolaos Toumbas \\ Department of Physics, University of Cyprus, P.O. Box 20537, 1678 Nicosia, Cyprus \\ Correspondence should be addressed to Constantinos Pallis; kpallis@gen.auth.gr
}

Received 29 December 2016; Revised 23 March 2017; Accepted 10 April 2017; Published 18 June 2017

Academic Editor: Elias C. Vagenas

Copyright (C) 2017 Constantinos Pallis and Nicolaos Toumbas. This is an open access article distributed under the Creative Commons Attribution License, which permits unrestricted use, distribution, and reproduction in any medium, provided the original work is properly cited. The publication of this article was funded by SCOAP ${ }^{3}$.

\begin{abstract}
We review the realization of Starobinsky-type inflation within induced-gravity supersymmetric (SUSY) and non-SUSY models. In both cases, inflation is in agreement with the current data and can be attained for sub-Planckian values of the inflation. The corresponding effective theories retain perturbative unitarity up to the Planck scale and the inflation mass is predicted to be $3 \cdot 10^{13} \mathrm{GeV}$. The supergravity embedding of these models is achieved by employing two gauge singlet chiral superfields, a superpotential that is uniquely determined by a continuous $R$ and a discrete $\mathbb{Z}_{n}$ symmetry and several (semi)logarithmic Kähler potentials that respect these symmetries. Checking various functional forms for the noninflation accompanying field in the Kähler potentials, we identify four cases which stabilize it without invoking higher order terms.
\end{abstract}

\section{Introduction}

The idea that the universe underwent a period of exponential expansion, called inflation [1-3], has proven useful not only for solving the horizon and flatness problems of standard cosmology but also for providing an explanation for the scale invariant perturbations, which are responsible for generating the observed anisotropies in the Cosmic Microwave Background $(\mathrm{CMB})$. One of the first incarnations of inflation is due to Starobinsky. To date, this attractive scenario remains predictive, since it passes successfully all the observational tests $[4,5]$. Starobinsky considered adding an $\mathscr{R}^{2}$ term, where $\mathscr{R}$ is the Ricci scalar, to the standard Einstein action in order to source inflation. Recall that gravity theories based on higher powers of $\mathscr{R}$ are equivalent to standard gravity theories with one additional scalar degree of freedom (see, e.g., $[6,7])$. As a result, Starobinsky inflation is equivalent to inflation driven by a scalar field with a suitable potential, so it admits several interesting realizations [8-29].

Following this route, we show in this work that inducedgravity inflation (IGI) [30-38] is effectively Starobinsky-like, reproducing the structure and the predictions of the original model. Within IGI, the inflation exhibits a strong coupling to $\mathscr{R}$ and the reduced Planck scale is dynamically generated through the vacuum expectation value (v.e.v.) of the inflation at the end of inflation. Therefore, the inflation acquires a Higgs-like behavior as in theories of induced gravity [36-42]. Apart from being compatible with data, the resulting theory respects perturbative unitarity up to the Planck scale [2931]. Therefore, no concerns about the validity of the corresponding effective theory arise. This is to be contrasted with models of nonminimal inflation (nMI) [43-54] based on a $\phi^{n}$ potential with negligible v.e.v. for the inflation $\phi$. Although these models yield similar observational predictions with the Starobinsky model, they admit an ultraviolet (UV) scale well below $m_{P}$ for $n>2$, leading to complications with naturalness [55-57].

Nonetheless, IGI allows us to embed Starobinsky inflation within $\mathcal{N}=1$ supergravity (SUGRA) in an elegant way. The embedding is achieved by incorporating two chiral superfields, a modulus-like field $T$ and a matter-like field $S$ appearing in the superpotential, $W$, as well as various Kähler potentials, $K$, consistent with an $R$ and discrete $\mathbb{Z}_{n}$ symmetries $[29,31,58]$; see also $[20-22,28,32]$. In some cases $[20,29,31,58]$, the employed $K$ 's parameterize specific Kähler manifolds, which appear in no-scale models [5961]. Moreover, this scheme ensures naturally a low enough reheating temperature, potentially consistent with the gravitino constraint $[29,62,63]$ if connected with a version of the Minimal SUSY Standard Model (MSSM). 
An important issue in embedding IGI in SUGRA is the stabilization of the matter-like field $S$. Indeed, when $K$ parameterizes the $S U(2,1) /(S U(2) \times U(1))$ Kähler manifold $[20,21]$, the inflationary trajectory turns out to be unstable with respect to the fluctuations of $S$. This difficulty can be overcome by adding a sufficiently large term $k_{S}|S|^{4}$, with $k_{S}>$ 0 and $\left|k_{S}\right| \sim 1$, in the logarithmic function appearing in $K$, as suggested in [64] for models of nonminimal (chaotic) inflation [47-49] and applied in [50-54, 65-70]. This solution, however, deforms slightly the Kähler manifold [71]. More importantly, it violates the predictability of Starobinsky inflation, since mixed terms $k_{S T}|S|^{2}|T|^{2}$ with $k_{S T} \geq 0.01$, which cannot be ignored (without tuning), have an estimable impact [31, 72-74] on the dynamics and the observables. Moreover, this solution becomes complicated when more than two fields are considered, since all quartic terms allowed by symmetries have to be considered, and the analysis of the stabilization mechanism becomes tedious (see, e.g., [31, 72$74]$ ). Alternatively, it was suggested to use a nilpotent superfield $S$ [75] or a charged field under a gauged $R$ symmetry [71].

In this review, we revisit the issue of stabilizing $S$, disallowing terms of the form $|S|^{2 m}, m>1$, without caring much about the structure of the Kähler manifold. Namely, we investigate systematically several functions $h_{i}\left(|S|^{2}\right.$ ) (with $i=1, \ldots, 11)$ that appear in the choices for $K$, and we find four acceptable forms that lead to the stabilization of $S$ during and after IGI. The output of this analysis is new, providing results that did not appear in the literature before. More specifically, we consider two principal classes of $K$ 's, $K_{3 i}$, and $K_{2 i}$, distinguished by whether $h_{i}$ and $T$ appear in the same logarithmic function. The resulting inflationary scenarios are almost indistinguishable. The case considered in [58] is included as one of the viable choices in $K_{2 i}$ class. Contrary to [58], we impose here the same $\mathbb{Z}_{n}$ symmetry on $W$ and $K$. Consequently, the relevant expressions for the mass spectrum and the inflationary observables get simplified considerably compared to those displayed in [58]. As in the non-SUSY case, IGI may be realized using sub-Planckian values for the initial (noncanonically normalized) inflation field. The radiative corrections remain under control and perturbative unitarity is not violated up to $m_{P}[31,58,76]$, consistently with the consideration of SUGRA as an effective theory.

Throughout this review we focus on the standard $\Lambda$ CDM cosmological model [4]. An alternative framework is provided by the running vacuum models [77-84] which turn out to yield a quality fit to observations, significantly better than that of $\Lambda C D M$. In this case, the acceleration of the universe, either during inflation or at late times, is not attributed to a scalar field but rather arises from the modification of the vacuum itself, which is dynamical. A SUGRA realization of Starobinsky inflation within this setting is obtained in [18].

The plan of this paper is as follows. In Section 2, we establish the realization of Starobinsky inflation as IGI in a non-SUSY framework. In Section 3 we introduce the formulation of IGI in SUGRA and revisit the issue of stabilizing the matter-like field $S$. The emerging inflationary models are analyzed in Section 4. Our conclusions are summarized in Section 5. Throughout, charge conjugation is denoted by a star $\left({ }^{*}\right)$, the symbol , $z$ as subscript denotes derivation with respect to $z$, and we use units where the reduced Planck scale, $m_{P}=2.43 \cdot 10^{18} \mathrm{GeV}$, is set equal to unity.

\section{Starobinsky Inflation from Induced Gravity}

We begin our presentation demonstrating the connection between $\mathscr{R}^{2}$ inflation and IGI. We first review the formulation of $\mathrm{nMI}$ in Section 2.1 and then proceed to describe the inflationary analysis in Section 2.2. Armed with these prerequisites, we present $\mathscr{R}^{2}$ inflation as a type of nMI in Section 2.3 and exhibit its connection with IGI in Section 2.4.

2.1. Coupling Nonminimally the Inflation to Gravity. We consider an inflation $\phi$ that is nonminimally coupled to the Ricci scalar $\mathscr{R}$, via a coupling function $f_{\mathscr{R}}(\phi)$. We denote the inflation potential by $V_{I}(\phi)$ and allow for a general kinetic function $f_{K}(\phi)$-in the cases of pure nMI $[33-35,45,46]$ $f_{K}=1$. The Jordan Frame (JF) action takes the form

$$
S=\int d^{4} x \sqrt{-\mathfrak{g}}\left(-\frac{1}{2} f_{\mathscr{R}} \mathscr{R}+\frac{1}{2} f_{K} g^{\mu \nu} \partial_{\mu} \phi \partial_{\nu} \phi-V_{I}(\phi)\right),
$$

where $\mathfrak{g}$ is the determinant of the Friedmann-RobertsonWalker metric, $g_{\mu \nu}$, with signature $(+,-,-,-)$. We require $\left\langle f_{\mathscr{R}}\right\rangle \simeq 1$ to ensure ordinary Einstein gravity at low energies.

By performing a conformal transformation [45] to the Einstein frame (EF), we write the action

$$
\mathrm{S}=\int d^{4} x \sqrt{-\widehat{\mathfrak{g}}}\left(-\frac{1}{2} \widehat{\mathscr{R}}+\frac{1}{2} \widehat{\mathfrak{g}}^{\mu \nu} \partial_{\mu} \widehat{\phi} \partial_{\nu} \widehat{\phi}-\widehat{V}_{I}(\widehat{\phi})\right),
$$

where a hat denotes an EF quantity. The EF metric is given by $\widehat{g}_{\mu \nu}=f_{\mathscr{R}} g_{\mu \nu}$, and the canonically normalized field, $\widehat{\phi}$, and its potential, $\widehat{V}_{I}$, are defined as follows:

$$
\begin{aligned}
& \text { (a) } \frac{d \widehat{\phi}}{d \phi}=J=\sqrt{\frac{f_{K}}{f_{\mathscr{R}}}+\frac{3}{2}\left(\frac{f_{\mathscr{R}, \phi}}{f_{\mathscr{R}}}\right)^{2}}, \\
& \text { (b) } \widehat{V}_{I}=\frac{V_{I}}{f_{\mathscr{R}}^{2}} .
\end{aligned}
$$

For $f_{\mathscr{R}} \gg f_{K}$, the coupling function $f_{\mathscr{R}}$ acquires a twofold role. On the one hand, it determines the relation between $\widehat{\phi}$ and $\phi$. On the other hand, it controls the shape of $\widehat{V}_{I}$, thus affecting the observational predictions; see below. The analysis of nMI can be performed in the EF, using the standard slow-roll approximation. It is [33-35] completely equivalent with the analysis in the JF. We just have to keep track the relation between $\widehat{\phi}$ and $\phi$.

2.2. Observational and Theoretical Constraints. A viable model of nMI must be compatible with a number of observational and theoretical requirements summarized in the following (cf. [85-88]).

(1) The number of e-foldings $\widehat{N}_{\star}$ that the scale $k_{\star}=$ $0.05 / \mathrm{Mpc}$ experiences during inflation must be large enough 
for the resolution of the horizon and flatness problems of the standard hot Big Bang model; that is, $[4,45]$

$$
\begin{aligned}
\widehat{N}_{\star}= & \int_{\widehat{\phi}_{f}}^{\widehat{\phi}_{\star}} d \widehat{\phi} \frac{\widehat{V}_{I}}{\widehat{V}_{I, \widehat{\phi}}}=\int_{\phi_{f}}^{\phi_{\star}} d \phi J^{2} \frac{\widehat{V}_{I}}{\widehat{V}_{I, \phi}} \\
\simeq & 61.7+\ln \frac{\widehat{V}_{I}\left(\phi_{\star}\right)^{1 / 2}}{\widehat{V}_{I}\left(\phi_{f}\right)^{1 / 3}}+\frac{1}{3} \ln T_{\mathrm{rh}} \\
& +\frac{1}{2} \ln \frac{f_{\mathscr{R}}\left(\phi_{\star}\right)}{f_{\mathscr{R}}\left(\phi_{f}\right)^{1 / 3}}
\end{aligned}
$$

$$
\max \left\{\widehat{\epsilon}\left(\phi_{f}\right),\left|\widehat{\eta}\left(\phi_{f}\right)\right|\right\}=1, \quad \text { where } \widehat{\epsilon}=\frac{1}{2}\left(\frac{\widehat{V}_{I, \widehat{\phi}}}{\widehat{V}_{I}}\right)^{2}=\frac{1}{2 J^{2}}\left(\frac{\widehat{V}_{I, \phi}}{\widehat{V}_{I}}\right)^{2}, \widehat{\eta}=\frac{\widehat{V}_{I, \widehat{\phi} \hat{\phi}}}{\widehat{V}_{I}}=\frac{1}{J^{2}}\left(\frac{\widehat{V}_{I, \phi \phi}}{\widehat{V}_{I}}-\frac{\widehat{V}_{I, \phi}}{\widehat{V}_{I}} \frac{J_{, \phi}}{J}\right)
$$

where $\phi_{\star}\left[\widehat{\phi}_{\star}\right]$ is the value of $\phi[\widehat{\phi}]$ when $k_{\star}$ crosses the inflationary horizon. In deriving the formula above (cf. [65-67]), we take into account an equation-of-state with parameter $w_{\text {rh }}=0$ [89], since $\widehat{V}_{I}$ can be well approximated by a quadratic potential for low values of $\phi$; see(20b), (32b), and (71b) below. Also $T_{\text {rh }}$ is the reheating temperature after nMI. We take a representative value $T_{\mathrm{rh}}=4.1 \cdot 10^{-10}$ throughout, which results in $\widehat{N}_{\star} \simeq 53$. The effective number of relativistic degrees of freedom at temperature $T_{\text {rh }}$ is taken $g_{\text {rh }}=107.75$ in accordance with the standard model spectrum. Lastly, $\phi_{f}\left[\hat{\phi}_{f}\right]$ is the value of $\phi[\widehat{\phi}]$ at the end of nMI, which in the slow-roll approximation can be obtained via the condition
Evidently nontrivial modifications of $f_{\mathscr{R}}$, and thus of $J$, may have a significant effect on the parameters above, modifying the inflationary observables.

(2) The amplitude $A_{s}$ of the power spectrum of the curvature perturbation generated by $\phi$ at $k_{\star}$ has to be consistent with the data [90]; that is,

$$
\begin{aligned}
\sqrt{A_{s}} & =\frac{1}{2 \sqrt{3} \pi} \frac{\widehat{V}_{I}\left(\widehat{\phi}_{\star}\right)^{3 / 2}}{\left|\widehat{V}_{I, \widehat{\phi}}\left(\widehat{\phi}_{\star}\right)\right|}=\frac{\left|J\left(\phi_{\star}\right)\right|}{2 \sqrt{3} \pi} \frac{\widehat{V}_{I}\left(\phi_{\star}\right)^{3 / 2}}{\left|\widehat{V}_{I, \phi}\left(\phi_{\star}\right)\right|} \\
& \simeq 4.627 \cdot 10^{-5} .
\end{aligned}
$$

As shown in Section 3.4, the remaining scalars in the SUGRA versions of $\mathrm{nMI}$ may be rendered heavy enough, so they do not contribute to $A_{s}$.

(3) The remaining inflationary observables (the spectral index $n_{s}$, its running $\alpha_{s}$, and the tensor-to-scalar ratio $r$ ) must be in agreement with the fitting of the Planck, Baryon Acoustic Oscillations (BAO) and BICEP2/Keck Array data $[4,5]$ with the $\Lambda \mathrm{CDM}+r$ model; that is,

$$
\begin{aligned}
& \text { (a) } n_{s}=0.968 \pm 0.009 \text {, } \\
& \text { (b) } r \leq 0.07,
\end{aligned}
$$

at the $95 \%$ confidence level (c.l.) with $\left|\alpha_{s}\right| \ll 0.01$. Although compatible with (7)(b), all data taken by the BICEP2/Keck Array CMB polarization experiments, up to the 2014 observational season (BK14) [5], seem to favor $r$ 's of the order of 0.01 , as the reported value is $0.028_{-0.025}^{+0.026}$ at the $68 \%$ c.l.. These inflationary observables are estimated through the relations:
(a) $n_{s}=1-6 \widehat{\epsilon}_{\star}+2 \widehat{\eta}_{\star}$,
(b) $\alpha_{s}=\frac{2}{3}\left(4 \widehat{\eta}_{\star}^{2}-\left(n_{s}-1\right)^{2}\right)-2 \widehat{\xi}_{\star}$,
(c) $r=16 \widehat{\epsilon}_{\star}$,

where $\widehat{\xi}=\widehat{V}_{I, \widehat{\phi}} \widehat{V}_{I, \widehat{\phi} \hat{\phi} \hat{\phi}} / \widehat{V}_{I}^{2}$ and the variables with subscript $\star$ are evaluated at $\phi_{\star}$.

(4) The effective theory describing nMI remains valid up to a UV cutoff scale $\Lambda_{\mathrm{UV}}$, which has to be large enough to ensure the stability of our inflationary solutions; that is,

$$
\begin{aligned}
& \text { (a) } \widehat{V}_{I}\left(\phi_{\star}\right)^{1 / 4} \leq \Lambda_{\mathrm{UV}}, \\
& \text { (b) } \phi_{\star} \leq \Lambda_{\mathrm{UV}} .
\end{aligned}
$$

As we show below, $\Lambda_{\mathrm{UV}} \simeq 1$ for the models analyzed in this work, contrary to the cases of pure nMI with large $f_{\mathscr{R}}$, where $\Lambda_{\mathrm{UV}} \ll 1$. The determination of $\Lambda_{\mathrm{UV}}$ is achieved expanding $\mathcal{S}$ in (2) about $\langle\phi\rangle$. Although these expansions are not strictly valid [57] during inflation, we take $\Lambda_{\mathrm{UV}}$ extracted this way to be the overall UV cutoff scale, since the reheating phase, realized via oscillations about $\langle\phi\rangle$, is a necessary stage of the inflationary dynamics.

2.3. From Nonminimal to $\mathscr{R}^{2}$ Inflation. $\mathscr{R}^{2}$ inflation can be viewed as a type of nMI, if we employ an auxiliary field $\phi$ with the following input ingredients:

$$
\begin{aligned}
f_{K} & =0, \\
f_{\mathscr{R}} & =1+4 c_{\mathscr{R}} \phi, \\
\widehat{V}_{I} & =\phi^{2} .
\end{aligned}
$$

Using the equation of motion for the auxiliary field, $\phi=c_{\mathscr{R}} \mathscr{R}$, we obtain the action of the original Starobinsky model (see, e.g., [71]):

$$
\mathrm{S}=\int d^{4} x \sqrt{-\mathfrak{g}}\left(-\frac{1}{2} \mathscr{R}+c_{\mathscr{R}}^{2} \mathscr{R}^{2}\right)
$$

As we can see from (10), the model has only one free parameter $\left(c_{\mathscr{R}}\right)$, enough to render it consistent with the observational data, ensuring at the same time perturbative 


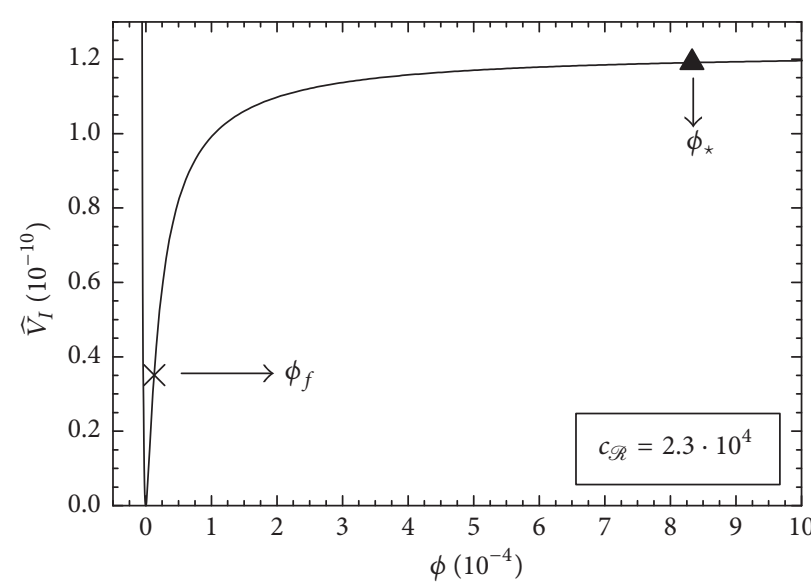

(a)

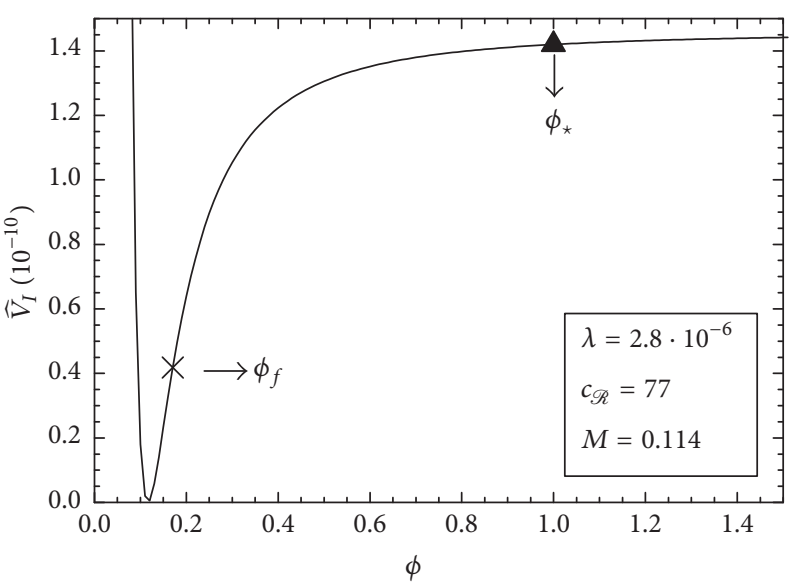

(b)

FIGURE 1: The inflationary potential $\widehat{V}_{I}$ as a function of $\phi$ for $\mathscr{R}^{2}$ inflation (a) and IGI with $\phi_{\star}=1(\mathrm{~b})$. Values corresponding to $\phi_{\star}$ and $\phi_{f}$ are also indicated.

unitarity up to the Planck scale. Using (10) and (3), we obtain the EF quantities:

$$
\begin{aligned}
& \text { (a) } J=2 \sqrt{6} \frac{c_{\mathscr{R}}}{f_{\mathscr{R}}}, \\
& \text { (b) } \widehat{V}_{I}=\frac{\phi^{2}}{f_{\mathscr{R}}^{2}} \simeq \frac{1}{16 c_{\mathscr{R}}^{2}} .
\end{aligned}
$$

For $c_{\mathscr{R}} \gg 1$, the plot of $\widehat{V}_{I}$ versus $\phi$ is depicted in Figure $1(\mathrm{a})$. An inflationary era can be supported since $\widehat{V}_{I}$ becomes flat enough. To examine further this possibility, we calculate the slow-roll parameters. Plugging (12) into (5) yields

$$
\begin{aligned}
& \widehat{\epsilon}=\frac{1}{12 c_{\mathscr{R}}^{2} \phi^{2}}, \\
& \widehat{\eta}=\frac{1-4 c_{\mathscr{R}} \phi}{12 c_{\mathscr{R}}^{2} \phi^{2}} .
\end{aligned}
$$

Notice that $\eta<0$ since $\widehat{V}_{I}$ is slightly concave downwards, as shown in Figure 1(a). The value of $\phi$ at the end of nMI is determined via (5), giving

$$
\begin{aligned}
& \phi_{f}=\max \left(\frac{1}{2 \sqrt{3} c_{\mathscr{R}}}, \frac{1}{6 c_{\mathscr{R}}}\right) \Longrightarrow \\
& \phi_{f}=\frac{1}{2 \sqrt{3} c_{\mathscr{R}}} .
\end{aligned}
$$

Under the assumption that $\phi_{f} \ll \phi_{\star}$, we can obtain a relation between $\widehat{N}_{\star}$ and $\phi_{\star}$ via (4)

$$
\widehat{N}_{\star} \simeq 3 c_{\mathscr{R}} \phi_{\star} .
$$

The precise value of $c_{\mathscr{R}}$ can be determined enforcing (6). Recalling that $\widehat{N}_{\star} \simeq 53$, we get

$$
\begin{aligned}
A_{s}^{1 / 2} & \simeq \frac{\widehat{N}_{\star}}{12 \sqrt{2} \pi c_{\mathscr{R}}}=4.627 \cdot 10^{-5} \Longrightarrow \\
c_{\mathscr{R}} & \simeq 2.3 \cdot 10^{4} .
\end{aligned}
$$

The resulting value of $c_{\mathscr{R}}$ is large enough so that

$$
\phi_{\star} \simeq \frac{\widehat{N}_{\star}}{3 c_{\mathscr{R}}} \simeq 8.3 \cdot 10^{-4} \ll 1
$$

consistently with (9)(b); see Figure 1(a). Impressively, the remaining observables turn out to be compatible with the observational data of (7). Indeed, inserting the above value of $\phi_{\star}$ into $(8)\left(\widehat{N}_{\star}=53\right)$, we get

$$
\begin{aligned}
n_{s} & \simeq \frac{\left(\widehat{N}_{\star}-3\right)\left(\widehat{N}_{\star}-1\right)}{\widehat{N}_{\star}^{2}} \simeq 1-\frac{2}{\widehat{N}_{\star}}-\frac{3}{\widehat{N}_{\star}^{2}} \simeq 0.961 ; \\
\alpha_{s} & \simeq-\frac{\left(\widehat{N}_{\star}-3\right)\left(4 \widehat{N}_{\star}+3\right)}{2 \widehat{N}_{\star}^{4}} \simeq-\frac{2}{\widehat{N}_{\star}^{2}}-\frac{15}{2 \widehat{N}_{\star}^{3}} \\
& \simeq-7.6 \cdot 10^{-4} ; \\
r & \simeq \frac{12}{\widehat{N}_{\star}^{2}} \simeq 4.2 \cdot 10^{-3} .
\end{aligned}
$$

Without the simplification of (15), we obtain numerically $n_{s}=$ $0.964, \alpha_{s}=-6.7 \cdot 10^{-4}$, and $r=3.7 \cdot 10^{-3}$. We see that $n_{s}$ turns out to be appreciably lower than unity thanks to the negative values of $\eta$; see (13). The mass of the inflation at the vacuum is

$$
\begin{aligned}
\widehat{m}_{\delta \phi} & =\left\langle\widehat{V}_{I, \widehat{\phi} \hat{\phi}}\right\rangle^{1 / 2}=\left\langle\frac{\widehat{V}_{I, \phi \phi}}{J^{2}}\right\rangle^{1 / 2}=\frac{1}{2 \sqrt{3} c_{\mathscr{R}}} \\
& \simeq 1.25 \cdot 10^{-5} \quad\left(\text { i.e. } 3 \cdot 10^{13} \mathrm{GeV}\right) .
\end{aligned}
$$

As we show below this value is salient future in all models of Starobinsky inflation.

Furthermore, the model provides an elegant solution to the unitarity problem [55-57], which plagues models of nMI with $f_{\mathscr{R}} \sim \phi^{n} \gg f_{K}, n>2$, and $f_{K}=1$. This stems from the fact that $\widehat{\phi}$ and $\phi$ do not coincide at the vacuum, as (12)(a) 
implies $\widehat{\phi}=\langle J\rangle \phi=2 \sqrt{3} c_{\mathscr{R}} \phi$. In fact, if we expand the second term in the right-hand side (r.h.s.) of (2) about $\langle\phi\rangle=0$, we find

$$
J^{2} \dot{\phi}^{2}=\left(1-2 \sqrt{\frac{2}{3}} \widehat{\phi}+2 \widehat{\phi}^{2}-\cdots\right) \dot{\hat{\phi}}^{2}
$$

Similarly, expanding $\widehat{V}_{I}$ in (12)(b), we obtain

$$
\widehat{V}_{I}=\frac{\widehat{\phi}^{2}}{24 c_{\mathscr{R}}^{2}}\left(1-2 \sqrt{\frac{2}{3}} \widehat{\phi}+2 \widehat{\phi}^{2}-\cdots\right) .
$$

Since the coefficients of the above series are of order unity, independent of $c_{\mathscr{R}}$, we infer that the model does not face any problem with perturbative unitarity up to the Planck scale.

2.4. Induced-Gravity Inflation. It would be certainly beneficial to realize the structure and the predictions of $\mathscr{R}^{2}$ inflation in a framework that deviates minimally from Einstein gravity, at least in the present cosmological era. To this extent, we incorporate the idea of induced gravity, according to which $m_{P}$ is generated dynamically $[41,42]$ via the v.e.v. of a scalar field $\phi$, driving a phase transition in the early universe. The simplest way to implement this scheme is to employ a doublewell potential for $\phi$; for scale invariant realizations of this idea, see $[39,40]$. On the other hand, an inflationary stage requires a sufficiently flat potential, as in (10). This can be achieved at large field values if we introduce a quadratic $f_{\mathscr{R}}$ [33-38]. More explicitly, IGI may be defined as nMI with the following input ingredients:

$$
\begin{aligned}
f_{K} & =1, \\
f_{\mathscr{R}} & =c_{\mathscr{R}} \phi^{2}, \\
V_{I} & =\frac{\lambda\left(\phi^{2}-M^{2}\right)^{2}}{4} .
\end{aligned}
$$

Given that $\langle\phi\rangle=M$, we recover Einstein gravity at the vacuum if

$$
\begin{aligned}
f_{\mathscr{R}}(\langle\phi\rangle) & =1 \Longrightarrow \\
M & =\frac{1}{\sqrt{c_{\mathscr{R}}}} .
\end{aligned}
$$

We see that in this model there is one additional free parameter, namely, $\lambda$ appearing in the potential, as compared to $\mathscr{R}^{2}$ model.

Equations (3) and (21) imply

$$
\begin{aligned}
& \text { (a) } J \simeq \frac{\sqrt{6}}{\phi} \\
& \text { (b) } \widehat{V}_{I}=\frac{\lambda f_{\phi}^{2}}{4 c_{\mathscr{R}}^{4} \phi^{4}} \simeq \frac{\lambda}{4 c_{\mathscr{R}}^{2}} \text { with } f_{\phi}=1-c_{\mathscr{R}} \phi^{2} .
\end{aligned}
$$

For $c_{\mathscr{R}} \gg 1$, the plot of $\widehat{V}_{I}$ versus $\phi$ is shown in Figure 1(b). As in $\mathscr{R}^{2}$ model, $\widehat{V}_{I}$ develops a plateau, so an inflationary stage can be realized. To check its robustness, we compute the slowroll parameters. Equations (5) and (23) give

$$
\begin{aligned}
& \widehat{\epsilon}=\frac{4}{3 f_{\phi}^{2}}, \\
& \widehat{\eta}=\frac{4\left(1+f_{\phi}\right)}{3 f_{\phi}^{2}} .
\end{aligned}
$$

IGI is terminated when $\phi=\phi_{f}$, determined by the condition

$$
\begin{aligned}
& \phi_{f}=\max \left(\sqrt{\frac{1+2 / \sqrt{3}}{c_{\mathscr{R}}}}, \sqrt{\frac{5}{3 c_{\mathscr{R}}}}\right) \Longrightarrow \\
& \phi_{f}=\sqrt{\frac{1+2 / \sqrt{3}}{c_{\mathscr{R}}}} .
\end{aligned}
$$

Under the assumption that $\phi_{f} \ll \phi_{\star}$, (4) implies the following relation between $\widehat{N}_{\star}$ and $\phi_{\star}$ :

$$
\begin{aligned}
\widehat{N}_{\star} & \simeq \frac{3 c_{\mathscr{R}} \phi_{\star}^{2}}{4} \Longrightarrow \\
\phi_{\star} & \simeq 2 \sqrt{\frac{\widehat{N}_{\star}}{3 c_{\mathscr{R}}}} \gg \phi_{f} .
\end{aligned}
$$

Imposing (9)(b) and setting $\widehat{N}_{\star} \simeq 53$, we derive a lower bound on $c_{\mathscr{R}}$ :

$$
\begin{aligned}
& \phi_{\star} \leq 1 \Longrightarrow \\
& c_{\mathscr{R}} \geq \frac{4 \widehat{N}_{\star}}{3} \simeq 71 .
\end{aligned}
$$

Contrary to $\mathscr{R}^{2}$ inflation, $c_{\mathscr{R}}$ does not control exclusively the normalization of (6), thanks to the presence of an extra factor of $\sqrt{\lambda}$. This is constrained to scale with $c_{\mathscr{R}}$. Indeed, we have

$$
\begin{array}{r}
A_{s}^{1 / 2} \simeq \frac{\sqrt{\lambda} \widehat{N}_{\star}}{6 \sqrt{2} \pi c_{\mathscr{R}}}=4.627 \cdot 10^{-5} \Longrightarrow c_{\mathscr{R}} \simeq 42969 \sqrt{\lambda} \\
\qquad \text { for } \widehat{N}_{\star} \simeq 53 .
\end{array}
$$

If, in addition, we impose the perturbative bound $\lambda \leq 3.5$, we end-up with following ranges:

$$
\begin{aligned}
77 & \lesssim c_{\mathscr{R}} \lesssim 8.5 \cdot 10^{4}, \\
2.8 \cdot 10^{-6} & \lesssim \lambda \lesssim 3.5,
\end{aligned}
$$

where the lower bounds on $c_{T}$ and $\lambda$ correspond to $\phi_{\star}=1$; see Figure 1(b). Within the allowed ranges, $\widehat{m}_{\delta \phi}$ remains constant, by virtue of (28). The mass turns out to be

$$
\widehat{m}_{\delta \phi}=\frac{\sqrt{\lambda}}{\sqrt{3} c_{\mathscr{R}}} \simeq 1.25 \cdot 10^{-5},
$$


essentially equal to that estimated in (19). Moreover, using (26) and (8), we extract the remaining observables

$$
\begin{aligned}
n_{s} & =\frac{\left(4 \widehat{N}_{\star}-15\right)\left(4 \widehat{N}_{\star}+1\right)}{\left(3-4 \widehat{N}_{\star}\right)^{2}} \simeq 1-\frac{2}{\widehat{N}_{\star}}-\frac{9}{2 \widehat{N}_{\star}^{2}} \\
& \simeq 0.961 ; \\
\alpha_{s} & =-\frac{128 \widehat{N}_{\star}\left(4 \widehat{N}_{\star}+9\right)}{\left(3-4 \widehat{N}_{\star}\right)^{4}} \simeq-\frac{2}{\widehat{N}_{\star}^{2}}-\frac{21}{2 \widehat{N}_{\star}^{3}} \\
& \simeq-7.7 \cdot 10^{-4} ; \\
r & =\frac{192}{\left(3-4 \widehat{N}_{\star}\right)^{2}} \simeq \frac{12}{\widehat{N}_{\star}^{2}} \simeq 4.4 \cdot 10^{-3} .
\end{aligned}
$$

Without making the approximation of (26), we obtain numerically $\left(n_{s}, \alpha_{s}, r\right)=\left(0.964,-6.6 \cdot 10^{-4}, 3.7 \cdot 10^{-3}\right)$. These results practically coincide with those of $\mathscr{R}^{2}$ inflation, given in (18a)-(18c), and they are in excellent agreement with the observational data presented in (7).

As in the previous section, the model retains perturbative unitarity up to $m_{P}$. To verify this, we first expand the second term in the r.h.s. of (1) about $\widehat{\delta \phi}=\phi-M \simeq 0$, with $J$ given by (23)(a). We find

$$
\begin{aligned}
& J^{2} \dot{\phi}^{2}=\left(1-\sqrt{\frac{2}{3}} \widehat{\delta \phi}+\frac{1}{2} \widehat{\delta \phi}^{2}-\cdots\right) \dot{\overrightarrow{\delta \phi}}^{2} \\
& \text { with } \widehat{\delta \phi} \simeq \sqrt{6 c_{\mathscr{R}}} \delta \phi .
\end{aligned}
$$

Expanding $\widehat{V}_{I}$ given by (23)(b), we get

$$
\widehat{V}_{I}=\frac{\lambda^{2}}{6 c_{\mathscr{R}}^{2}} \widehat{\delta \phi}^{2}\left(1-\sqrt{\frac{3}{2}} \widehat{\delta \phi}+\frac{25}{24} \widehat{\delta \phi}^{2}-\cdots\right) .
$$

Therefore, $\Lambda_{\mathrm{UV}}=1$ as for $\mathscr{R}^{2}$ inflation. Practically identical results can be obtained if we replace the quadratic exponents in (21) with $n \geq 3$ as first pointed out in [30]. This generalization can be elegantly performed [31, 32] within SUGRA, as we review below.

\section{Induced-Gravity Inflation in SUGRA}

In Section 3.1, we present the general SUGRA setting, where IGI is embedded. Then, in Section 3.2, we examine a variety of Kähler potentials, which lead to the desired inflationary potential; see Section 3.3. We check the stability of the inflationary trajectory in Section 3.4.

3.1. The General Set-Up. To realize IGI within SUGRA [29, 31, $32,58]$, we must use two gauge singlet chiral superfields $z^{\alpha}$, with $z^{1}=T$ and $z^{2}=S$ being the inflation and a "stabilizer" superfield, respectively. Throughout this work, the complex scalar fields $z^{\alpha}$ are denoted by the same superfield symbol. The EF effective action is written as follows [47-49]:

$$
\mathrm{S}=\int d^{4} x \sqrt{-\widehat{\mathfrak{g}}}\left(-\frac{1}{2} \widehat{\mathscr{R}}+K_{\alpha \bar{\beta}} \widehat{\mathfrak{g}}^{\mu \nu} \partial_{\mu} z^{\alpha} \partial_{\gamma} z^{* \bar{\beta}}-\widehat{V}\right),
$$

where $K_{\alpha \bar{\beta}}=K_{, z^{\alpha} z^{* \bar{\beta}}}$ is the Kähler metric and $K^{\alpha \bar{\beta}}$ its inverse $\left(K^{\alpha \bar{\beta}} K_{\bar{\beta} \gamma}=\delta_{\gamma}^{\alpha}\right) \cdot \widehat{V}$ is the EF F-term SUGRA potential, given in terms of the Kähler potential $K$ and the superpotential $W$ by the following expression:

$$
\begin{array}{r}
\widehat{V}=e^{K}\left(K^{\alpha \bar{\beta}} D_{\alpha} W D_{\bar{\beta}}^{*} W^{*}-3|W|^{2}\right) \\
\text { with } D_{\alpha} W=W_{, z^{\alpha}}+K_{, z^{\alpha}} W .
\end{array}
$$

Conformally transforming to the JF with $f_{\mathscr{R}}=-\Omega / N$, where $N$ is a dimensionless positive parameter, $\mathrm{S}$ takes the form

$$
\begin{aligned}
\mathrm{S} & =\int d^{4} x \sqrt{-\mathfrak{g}}\left(\frac{\Omega}{2 N} \mathscr{R}+\frac{3}{4 N \Omega} \partial_{\mu} \Omega \partial^{\mu} \Omega\right. \\
& \left.-\frac{1}{N} \Omega K_{\alpha \bar{\beta}} \partial_{\mu} z^{\alpha} \partial^{\mu} z^{* \bar{\beta}}-V\right) \quad \text { with } V=\frac{\Omega^{2}}{N^{2}} \widehat{V} .
\end{aligned}
$$

Note that $N=3$ reproduces the standard set-up [47-49]. Let us also relate $\Omega$ and $K$ by

$$
\begin{aligned}
-\frac{\Omega}{N} & =e^{-K / N} \Longrightarrow \\
K & =-N \ln \left(-\frac{\Omega}{N}\right) .
\end{aligned}
$$

Then taking into account the definition [47-49] of the purely bosonic part of the auxiliary field when on shell,

$$
\mathscr{A}_{\mu}=\frac{i\left(K_{\alpha} \partial_{\mu} z^{\alpha}-K_{\bar{\alpha}} \partial_{\mu} z^{* \bar{\alpha}}\right)}{6},
$$

we arrive at the following action:

$$
\begin{aligned}
S & =\int d^{4} x \sqrt{-\mathfrak{g}}\left(\frac{\Omega}{2 N} \mathscr{R}\right. \\
& +\left(\Omega_{\alpha \bar{\beta}}+\frac{3-N}{N} \frac{\Omega_{\alpha} \Omega_{\bar{\beta}}}{\Omega}\right) \partial_{\mu} z^{\alpha} \partial^{\mu} z^{* \bar{\beta}} \\
& \left.-\frac{27}{N^{3}} \Omega_{A_{\mu}} \mathscr{A}^{\mu}-V\right) .
\end{aligned}
$$

By virtue of (35), $\mathscr{A}_{\mu}$ takes the form

$$
\mathscr{A}_{\mu}=-\frac{i N\left(\Omega_{\alpha} \partial_{\mu} z^{\alpha}-\Omega_{\bar{\alpha}} \partial_{\mu} z^{* \bar{\alpha}}\right)}{6 \Omega}
$$

with $\Omega_{\alpha}=\Omega_{, z^{\alpha}}$ and $\Omega_{\bar{\alpha}}=\Omega_{, z^{*} \bar{\alpha}}$. As can be seen from (37a), $-\Omega / N$ introduces a nonminimal coupling of the scalar fields to gravity. Ordinary Einstein gravity is recovered at the vacuum when

$$
-\frac{\langle\Omega\rangle}{N} \simeq 1 .
$$


TABLE 1: Definition of the various $h_{i}(X)$ 's, $h_{i}^{\prime \prime}(0)=d^{2} h_{i}(0) / d X^{2}$ and masses squared of the fluctuations of $s$ and $\bar{s}$ along the inflationary trajectory in (46) for $K=K_{3 i}$ and $K_{2 i}$.

\begin{tabular}{lcccc}
\hline$i$ & $h_{i}(X)$ & $h_{i}^{\prime \prime}(0)$ & $\widehat{m}_{s}^{2} / \widehat{H}_{I}^{2}$ & $K=K_{2 i}$ \\
\hline 1 & $X$ & 0 & $-2+2^{n} / f_{\phi}^{2}$ & $3 \cdot 2^{n-1} / f_{\phi}^{2}$ \\
\hline 2 & $e^{X}-1$ & 1 & $2^{(4-n) / 2} c_{T} \phi^{n}-2+2^{n} / f_{\phi}^{2}$ & $-6+3 \cdot 2^{n-1} / f_{\phi}^{2}$ \\
3 & $\ln (X+1)$ & -1 & $-2\left(1+2^{1-n / 2} c_{T} \phi^{n}\right)$ & $6\left(1+2^{n-1} / f_{\phi}^{2}\right)$ \\
\hline 4 & $-\cos (\arcsin 1+X)$ & 0 & $-2\left(1-2^{n-1} / f_{\phi}^{2}\right)$ & $3 \cdot 2^{n-1} / f_{\phi}^{2}$ \\
5 & $\sin (\arccos 1+X)$ & 0 & $-2\left(1-2^{n-1} / f_{\phi}^{2}\right)$ & $3 \cdot 2^{n-1} / f_{\phi}^{2}$ \\
6 & $\tan (X)$ & 0 & $-2\left(1-2^{n-1} / f_{\phi}^{2}\right)$ & $3 \cdot 2^{n-1} / f_{\phi}^{2}$ \\
7 & $-\cot (\arcsin 1+X)$ & 0 & $-2\left(1-2^{n-1} / f_{\phi}^{2}\right)$ & $3 \cdot 2^{n-1} / f_{\phi}^{2}$ \\
\hline 8 & $\cosh (\arcsin 1+X)-\sqrt{2}$ & $\sqrt{2}$ & $2^{(5-n) / 2} c_{T} \phi^{n}-2+2^{n} / f_{\phi}^{2}$ & $3 \cdot 2^{n-1} / f_{\phi}^{2}-6 \sqrt{2}$ \\
9 & $\sinh (X)$ & 0 & $-2\left(1-2^{n-1} / f_{\phi}^{2}\right)$ & $3 \cdot 2^{n-1} / f_{\phi}^{2}$ \\
10 & $\tanh (X)$ & 0 & $-2\left(1-2^{n-1} / f_{\phi}^{2}\right)$ & $3 \cdot 2^{n-1} / f_{\phi}^{2}$ \\
11 & $-\operatorname{coth}(\operatorname{arcsinh} 1+X)+\sqrt{2}$ & $-2 \sqrt{2}$ & $2^{n} / f_{\phi}^{2}-2^{(7-n) / 2} c_{T} \phi^{n}-2$ & $3 \cdot 2^{n-1} / f_{\phi}^{2}+12 \sqrt{2}$ \\
\hline
\end{tabular}

Starting with the JF action in (37a), we seek to realize IGI, postulating the invariance of $\Omega$ under the action of a global $\mathbb{Z}_{n}$ discrete symmetry. With $S$ stabilized at the origin, we write

$$
\begin{aligned}
& -\frac{\Omega}{N}=\Omega_{H}(T)+\Omega_{H}^{*}\left(T^{*}\right) \\
& \quad \text { with } \Omega_{H}(T)=c_{T} T^{n}+\sum_{k=2}^{\infty} \lambda_{k} T^{k n},
\end{aligned}
$$

where $k$ is a positive integer. If $T \leq 1$ during IGI and assuming that $\lambda_{k}$ 's are relatively small, the contributions of the higher powers of $T$ in the expression above are small, and these can be dropped. As we verify later, this can be achieved when the coefficient $c_{T}$ is large enough. Equivalently, we may rescale the inflation, setting $T \rightarrow \widetilde{T}=c_{T}{ }^{1 / n} T$. Then the coefficients $\lambda_{k}$ of the higher powers in the expression of $\Omega$ get suppressed by factors of $c_{T}^{-k}$. Thus, $\mathbb{Z}_{n}$ and the requirement $T \leq 1$ determine the form of $\Omega$, avoiding a severe tuning of the coefficients $\lambda_{k}$. Under these assumptions, $K$ in (35) takes the form

$$
K_{0}=-N \ln \left(f(T)+f^{*}\left(T^{*}\right)\right) \quad \text { with } f(T) \simeq c_{T} T^{n},
$$

where $S$ is assumed to be stabilized at the origin.

Equations (35) and (38) require that $T$ and $S$ acquire the following v.e.v.s:

$$
\begin{aligned}
& \langle T\rangle \simeq \frac{1}{\left(2 c_{T}\right)^{1 / n}}, \\
& \langle S\rangle=0 .
\end{aligned}
$$

These v.e.v.s can be achieved, if we choose the following superpotential $[31,32]$ :

$$
W=\lambda S\left(T^{n}-\frac{1}{2 c_{T}}\right) .
$$

Indeed the corresponding F-term SUSY potential, $V_{\text {SUSY }}$, is found to be

$$
V_{\text {SUSY }}=\lambda^{2}\left|T^{n}-\frac{1}{2 c_{T}}\right|^{2}+\lambda^{2} n^{2}\left|S T^{n-1}\right|^{2}
$$

and is minimized by the field configuration in (21).
As emphasized in $[29,31,58]$, the forms of $W$ and $\Omega_{H}$ can be uniquely determined if we limit ourselves to integer values for $n$ (with $n>1$ ) and $T \leq 1$ and impose two symmetries:

(i) An $R$ symmetry under which $S$ and $T$ have charges 1 and 0 , respectively.

(ii) A discrete symmetry $\mathbb{Z}_{n}$ under which only $T$ is charged.

For simplicity we assume here that both $W$ and $\Omega_{H}$ respect the same $\mathbb{Z}_{n}$, contrary to the situation in [58]. This assumption simplifies significantly the formulae in Sections 3.3 and 3.4. Note, finally, that the selected $\Omega$ in (39) does not contribute in the kinetic term involving $\Omega_{T T^{*}}$ in (37a). We expect that our findings are essentially unaltered even if we include in the r.h.s. of (39) a term $-\left(T-T^{*}\right)^{2} / 2 N$ [32] or $-|T|^{2} / N$ [31] which yields $\Omega_{T T^{*}}=1 \ll c_{T}$; the former choice, though, violates $\mathbb{Z}_{n}$ symmetry above.

3.2. Proposed Kähler Potentials. It is obvious from the considerations above that the stabilization of $S$ at zero during and after IGI is of crucial importance for the viability of our scenario. This key issue can be addressed if we specify the dependence of the Kähler potential on $S$. We distinguish the following basic cases:

$$
\begin{aligned}
& K_{3 i}=-n_{3} \ln \left(f(T)+f^{*}\left(T^{*}\right)+h_{i}(X)\right), \\
& K_{2 i}=-n_{2} \ln \left(f(T)+f^{*}\left(T^{*}\right)\right)+h_{i}(X),
\end{aligned}
$$

where the various choices $h_{i}, i=1, \ldots, 11$, are specified in Table 1 and $X$ is defined as follows:

$$
X=\left\{\begin{array}{cc}
-\frac{|S|^{2}}{n_{3}} & \text { for } K=K_{3 i} \\
|S|^{2} & \text { for } K=K_{2 i} .
\end{array}\right.
$$

As shown in Table 1 we consider exponential, logarithmic, trigonometric, and hyperbolic functions. Note that $K_{31}$ and 
$K_{21}$ parameterize $S U(2,1) / S U(2) \times U(1)$ and $S U(1,1) / U(1) \times$ $U(1)$ Kähler manifolds, respectively, whereas $K_{23}$ parameterizes the $S U(1,1) / U(1) \times S U(2) / U(1)$ Kähler manifold; see [58].

To show that the proposed $K$ 's are suitable for IGI, we have to verify that they reproduce $\widehat{V}_{I}$ in (23)(b) when $n=2$, and they ensure the stability of $S$ at zero. These requirements are checked in the following two sections.

3.3. Derivation of the Inflationary Potential. Substituting $W$ of (42) and a choice for $K$ in (44) (with the $h_{i}$ 's given in Table 1 ) into (33b), we obtain a potential suitable for IGI. The inflationary trajectory is defined by the constraints

$$
\begin{aligned}
S & =T-T^{*}=0, \\
\text { or } s & =\bar{s}=\theta=0,
\end{aligned}
$$

where we have expanded $T$ and $S$ in real and imaginary parts as follows:

$$
\begin{aligned}
& T=\frac{\phi}{\sqrt{2}} e^{i \theta}, \\
& S=\frac{s+i \bar{s}}{\sqrt{2}} .
\end{aligned}
$$

Along the path of (46), $\widehat{V}$ reads

$$
\widehat{V}_{I}=\widehat{V}(\theta=s=\bar{s}=0)=e^{K} K^{S S^{*}}\left|W_{, S}\right|^{2} .
$$

From (42) we get $W_{S}=f-1 / 2$. Also, (44) yields

$$
e^{K}= \begin{cases}\left(2 f+h_{i}(0)\right)^{-n_{3}} & \text { for } K=K_{3 i} \\ \frac{e^{h_{i}(0)}}{(2 f)^{n_{2}}} & \text { for } K=K_{2 i},\end{cases}
$$

where we take into account that $f(T)=f^{*}\left(T^{*}\right)$ along the path of (46). Moreover, $K^{S S^{*}}=1 / K_{S S^{*}}$ can be obtained from the Kähler metric, which is given by

$$
\begin{aligned}
& \left(K_{\alpha \bar{\beta}}\right)=\operatorname{diag}\left(K_{T T^{*}}, K_{S S^{*}}\right) \\
& \quad= \begin{cases}\operatorname{diag}\left(\frac{n_{3} n^{2}}{2 \phi^{2}}, \frac{h_{i}^{\prime}(0)}{\left(2 f+h_{i}(0)\right)}\right) & \text { for } K=K_{3 i} \\
\operatorname{diag}\left(\frac{n_{2} n^{2}}{2 \phi^{2}}, h_{i}^{\prime}(0)\right) & \text { for } K=K_{2 i},\end{cases}
\end{aligned}
$$

where a prime denotes a derivative with respect to $X$. Note that $K_{T T^{*}}$ for $K=K_{2 i}$ (and $S \neq 0$ ) does not involve the field $S$ in its denominator, and so no geometric destabilization [91] can be activated, contrary to the $K=K_{3 i}$ case. Inserting $W_{\text {, }}$ and the results of (49) and (50) into (33b), we obtain

$$
\begin{aligned}
\widehat{V}_{I}= & \frac{\lambda^{2}(1-2 f)^{2}}{c_{T}^{2}} \\
& \cdot \begin{cases}\frac{\left(2 f+h_{i}(0)\right)^{1-n_{3}}}{h_{i}^{\prime}(0)} & \text { for } K=K_{3 i} \\
\frac{e^{h_{i}(0)}}{(2 f)^{n_{2}} h_{i}^{\prime}(0)} & \text { for } K=K_{2 i} .\end{cases}
\end{aligned}
$$

Recall that $f \sim \phi^{n}$; see (40). Then $\widehat{V}_{I}$ develops a plateau, with almost constant potential energy density, for $c_{T} \gg 1$ and $\phi<1$ (or $c_{T}=1$ and $\phi \gg 1$ ), if we impose the following conditions:

$$
\begin{aligned}
2 n= & \left\{\begin{array}{ll}
n\left(n_{3}-1\right) & \text { for } K=K_{3 i} \\
n n_{2} & \text { for } K=K_{2 i}
\end{array} \Longrightarrow\right. \\
& \begin{cases}n_{3}=3 & \text { for } K=K_{3 i} \\
n_{2}=2 & \text { for } K=K_{2 i} .\end{cases}
\end{aligned}
$$

This empirical criterion is very precise since the data on $n_{s}$ allows only tiny (of order 0.001) deviations [28]. Actually, the requirement $c_{T} \gg 1$ and the synergy between the exponents in $W$ and $K$ 's assist us to tame the well-known $\eta$ problem within SUGRA with a mild tuning. If we insert (52) into (51) and compare the result for $n=2$ with (23)(b) (replacing also $\lambda^{2}$ with $\lambda$ ), we see that the two expressions coincide, if we set

$$
\begin{aligned}
& h_{i}(0)=0, \\
& h_{i}^{\prime}(0)=1 .
\end{aligned}
$$

As we can easily verify the selected $h_{i}$ in Table 1 satisfy these conditions. Consequently, $\widehat{V}_{I}$ in (51) and the corresponding Hubble parameter $\widehat{H}_{I}$ take their final form:

$$
\begin{aligned}
& \text { (a) } \widehat{V}_{I}=\frac{\lambda^{2} f_{\phi}^{2}}{4 c_{T}^{4} \phi^{2 n}}, \\
& \text { (b) } \widehat{H}_{I}=\frac{\widehat{V}_{I}^{1 / 2}}{\sqrt{3}}=\frac{\lambda f_{\phi}}{2 \sqrt{3} c_{T}^{2} \phi^{n}},
\end{aligned}
$$

with $f_{\phi}=2^{n / 2-1}-c_{T} \phi^{n}<0$ reducing to that defined in $(23)(\mathrm{b})$. Based on these expressions, we investigate in Section 4 the dynamics and predictions of IGI.

3.4. Stability of the Inflationary Trajectory. We proceed to check the stability of the direction in (46) with respect to the fluctuations of the various fields. To this end, we have to examine the validity of the extremum and minimum conditions; that is,
(a) $\left.\frac{\partial \widehat{V}_{I}}{\partial \hat{\bar{z}}^{\alpha}}\right|_{s=\bar{s}=\theta=0}=0$,
(b) $\widehat{m}_{\bar{z}^{\alpha}}^{2}>0$ with $\bar{z}^{\alpha}=\theta, s, \bar{s}$. 
TABLE 2: Mass-squared spectrum for $K=K_{3 i}$ and $K_{2 i}$ along the inflationary trajectory in (46).

\begin{tabular}{|c|c|c|c|c|}
\hline \multirow{2}{*}{ Fields } & \multirow{2}{*}{ Eigenstates } & \multicolumn{3}{|c|}{ Masses squared } \\
\hline & & & $K=K_{3 i}$ & $K=K_{2 i}$ \\
\hline 1 real scalar & $\widehat{\theta}$ & $\widehat{m}_{\theta}^{2} / \widehat{H}_{I}^{2}$ & $4\left(2^{n-2}-c_{T} \phi^{n} f_{\phi}\right) / f_{\phi}^{2}$ & $6\left(2^{n-2}-c_{T} \phi^{n} f_{\phi}\right) / f_{\phi}^{2}$ \\
\hline 1 complex & $\widehat{s}, \widehat{\bar{s}}$ & $\widehat{m}_{s}^{2} / \widehat{H}_{I}^{2}$ & $2^{n} / f_{\phi}^{2}-2$ & $3 \cdot 2^{n-1} / f_{\phi}^{2}$ \\
\hline scalar & & & $+2^{2-n / 2} c_{T} \phi^{n} h_{i}^{\prime \prime}(0)$ & $-6 h_{i}^{\prime \prime}(0)$ \\
\hline 2 Weyl spinors & $\widehat{\psi}_{ \pm}$ & $\widehat{m}_{\psi \pm}^{2} / \widehat{H}_{I}^{2}$ & $2^{n} / f_{\phi}^{2}$ & $6 \cdot 2^{n-3} / f_{\phi}^{2}$ \\
\hline
\end{tabular}

Here $\widehat{m}_{\bar{z}^{\alpha}}^{2}$ are the eigenvalues of the mass matrix with elements

$$
\widehat{M}_{\alpha \beta}^{2}=\left.\frac{\partial^{2} \widehat{V}_{I}}{\partial \hat{\bar{z}}^{\alpha} \partial \hat{\bar{z}}^{\beta}}\right|_{s=\bar{s}=\theta=0} \quad \text { with } \bar{z}^{\alpha}=\theta, s, \bar{s}
$$

and a hat denotes the EF canonically normalized field. The canonically normalized fields can be determined if we bring the kinetic terms of the various scalars in (33a) into the following form:

$$
K_{\alpha \bar{\beta}} \dot{z}^{\alpha} \dot{z}^{* \bar{\beta}}=\frac{1}{2}\left(\dot{\hat{\phi}}^{2}+\dot{\hat{\theta}}^{2}\right)+\frac{1}{2}\left(\dot{\hat{\mathcal{s}}}^{2}+\dot{\overline{\bar{s}}}^{2}\right),
$$

where a dot denotes a derivative with respect to the JF cosmic time. Then the hatted fields are defined as follows:

$$
\begin{aligned}
\frac{d \widehat{\phi}}{d \phi} & =J=\sqrt{K_{T T^{*}},} \\
\widehat{\theta} & =\frac{J \theta}{\phi}, \\
(\widehat{s}, \widehat{\bar{s}}) & =\sqrt{K_{S S^{*}}}(s, \bar{s}),
\end{aligned}
$$

where by virtue of (52) and (53), the Kähler metric of (50) reads

$$
\begin{aligned}
\left(K_{\alpha \bar{\beta}}\right)= & \operatorname{diag}\left(K_{T T^{*}}, K_{S S^{*}}\right) \\
& = \begin{cases}\left(\frac{3 n^{2}}{2 \phi^{2}}, \frac{2^{n / 2-1}}{c_{T} \phi^{n}}\right) & \text { for } K=K_{3 i} \\
\left(\frac{n^{2}}{\phi^{2}}, 1\right) & \text { for } K=K_{2 i} .\end{cases}
\end{aligned}
$$

Note that the spinor components $\psi_{T}$ and $\psi_{S}$ of the superfields $T$ and $S$ are normalized in a similar way; that is, $\widehat{\psi}_{T}=\sqrt{K_{T T *}} \psi_{\Phi}$ and $\widehat{\psi}_{S}=\sqrt{K_{S S^{*}}} \psi_{S}$. In practice, we have to make sure that all the $\widehat{m}_{\bar{z}^{\alpha}}^{2}$ 's are not only positive, but also greater than $\widehat{H}_{I}^{2}$ during the last 50-60 e-foldings of IGI. This guarantees that the observed curvature perturbation is generated solely by $\phi$, as assumed in (6). Nonetheless, two-field inflationary models which interpolate between the Starobinsky and the quadratic model have been analyzed in [92-95]. Due to the large effective masses that the scalars acquire during IGI, they enter a phase of damped oscillations about zero. As a consequence, $\phi$ dependence in their normalization (see (57b)) does not affect their dynamics.

We can readily verify that $(55)(a)$ is satisfied for all the three $\bar{z}^{\alpha}$ s. Regarding (55)(b), we diagonalize $\widehat{M}_{\alpha \beta}^{2}$ (56) and we obtain the scalar mass spectrum along the trajectory of (46). Our results are listed in Table 1 together with the masses squared $\widehat{m}_{\psi \pm}^{2}$ of the chiral fermion eigenstates $\widehat{\psi}_{ \pm}=\left(\widehat{\psi}_{T} \pm\right.$ $\left.\widehat{\psi}_{S}\right) / \sqrt{2}$. From these results, we deduce the following:

(i) For both classes of $K$ 's in (44), (55)(b) is satisfied for the fluctuations of $\widehat{\theta}$; that is, $\widehat{m}_{\theta}^{2}>0$, since $f_{\phi}<0$. Moreover, $\widehat{m}_{\theta}^{2} \gg \widehat{H}_{I}^{2}$ because $c_{T} \gg 1$.

(ii) When $K=K_{3 i}$ and $h_{i}^{\prime \prime}(0)=0$, we obtain $\widehat{m}_{s}^{2}<0$. This occurs for $i=1,4, \ldots, 7,9$ and 10, as shown in Table 1 . For $i=1$, our result reproduces those of similar models [31, 47-52, 68-70]. The stability problem can be cured if we include in $K_{3 i}$ a higher order term of the form $k_{S}|S|^{4}$ with $k_{S} \sim 1$, or assuming that $S^{2}=0$ [75]. However, a probably simpler solution arises if we take into account the results accumulated in Table 2. It is clear that the condition $\widehat{m}_{s}^{2}>\widehat{H}_{I}^{2}$ can be satisfied when $h_{i}^{\prime \prime}(0)>0$ with $\left|h_{i}^{\prime \prime}(0)\right| \geq 1$. From Table 1 , we see that this is the case for $i=2$ and 8 .

(iii) When $K=K_{2 i}$ and $h_{i}^{\prime \prime}(0)=0$, we obtain $\widehat{m}_{s}^{2}>0$, but $\widehat{m}_{s}^{2}<\widehat{H}_{I}^{2}$. Therefore, $S$ may seed inflationary perturbations, leading possibly to large non-Gaussianities in the CMB, contrary to observations. From the results listed in Table 2, we see that the condition $\widehat{m}_{s}^{2} \gg \widehat{H}_{I}^{2}$ requires $h_{i}^{\prime \prime}(0)<0$ with $\left|h_{i}^{\prime \prime}(0)\right| \geq 1$. This occurs for $i=3$ and 11. The former case was examined in [58].

To highlight further the stabilization of $S$ during and after IGI we present in Figure $2 \widehat{m}_{s}^{2} / \widehat{H}_{I}^{2}$ as a function of $\phi$ for the various acceptable $K$ 's identified above. In particular, we fix $n=2$ and $\phi_{\star}=1$, setting $K=K_{32}$ or $K=K_{38}$ in Figure 2(a) and $K=K_{23}$ or $K=K_{2,11}$ in Figure 2(b). The parameters of the models $\left(\lambda\right.$ and $c_{T}$ ) corresponding to these choices are listed in third and fifth rows of Table 3. Evidently $\widehat{m}_{s}^{2} / \widehat{H}_{I}^{2}$ remain larger than unity for $\phi_{f} \leq \phi \leq \phi_{\star}$, where $\phi_{\star}$ and $\phi_{f}$ are also depicted. However, in Figure 2(b) $\widehat{m}_{s}^{2} / \widehat{H}_{I}^{2}$ exhibits a constant behavior and increases sharply as $\phi$ decreases below 0.2. On the contrary, $\widehat{m}_{s}^{2} / \widehat{H}_{I}^{2}$ in Figure 2(a) is an increasing function of $\phi$ for $\phi \gtrsim 0.2$, with a clear minimum at $\phi \simeq 0.2$. For $\phi \lesssim 0.2, \widehat{m}_{s}^{2} / \widehat{H}_{I}^{2}$ increases drastically as in Figure 2(b) too.

Employing the well-known Coleman-Weinberg formula [96], we find from the derived mass spectrum (see Table 1) 


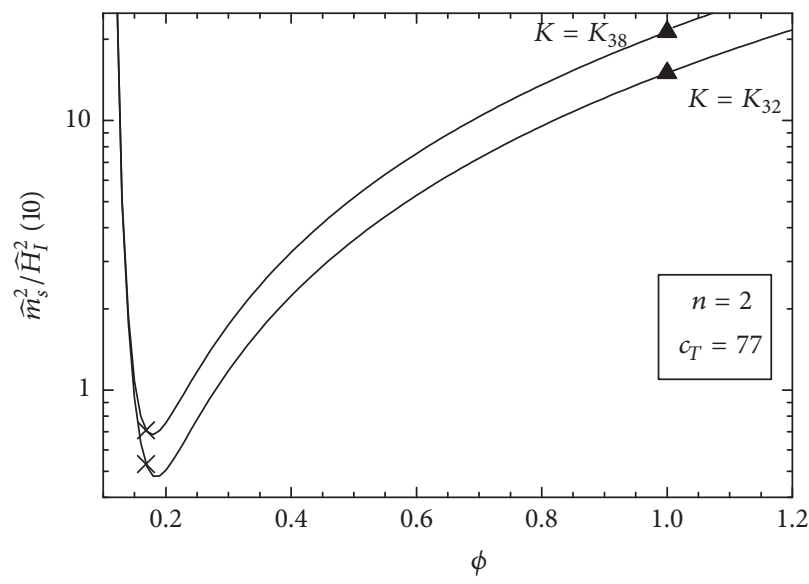

$\Delta \phi_{\star}$

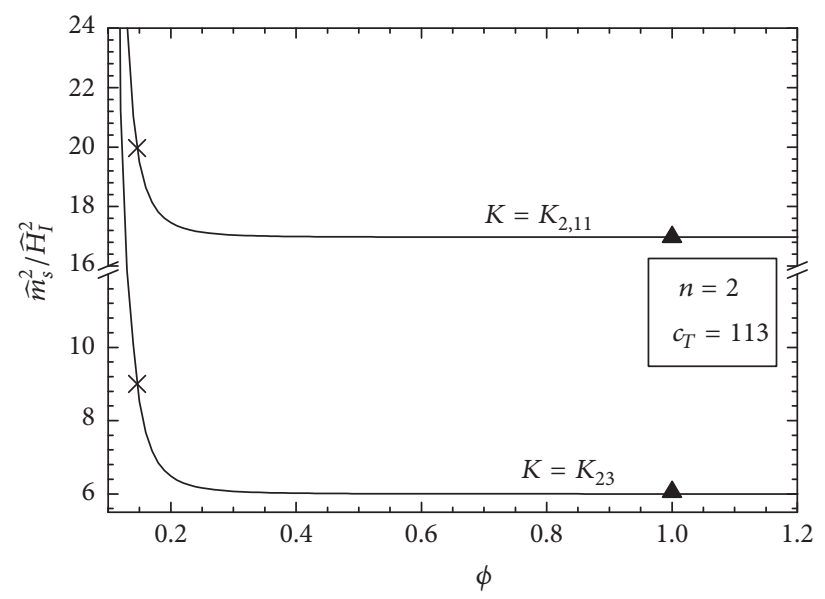

$\Delta \phi_{\star}$

$\times \phi_{f}$

(a)

(b)

Figure 2: The ratio $\widehat{m}_{s}^{2} / \widehat{H}_{I}^{2}$ as a function of $\phi$ for $n=2$ and $\phi_{\star}=1$. We set (a) $K=K_{32}$ or $K=K_{38}$ and (b) $K=K_{23}$ or $K=K_{2,11}$. The values corresponding to $\phi_{\star}$ and $\phi_{f}$ are also depicted.

the one-loop radiative corrections, $\Delta \widehat{V}_{I}$, to $\widehat{V}_{I}$, depending on renormalization group mass scale $\Lambda$. It can be verified that our results are insensitive to $\Delta \widehat{V}_{I}$, provided that $\Lambda$ is determined by requiring $\Delta \widehat{V}_{I}\left(\phi_{\star}\right)=0$ or $\Delta \widehat{V}_{I}\left(\phi_{f}\right)=0$. A possible dependence of the results on the choice of $\Lambda$ is totally avoided [31] thanks to the smallness of $\Delta \widehat{V}_{I}$, for $\Lambda \simeq(1-1.8) \cdot 10^{-5}$; see Section 4.2 too. These conclusions hold even for $\phi>1$. Therefore, our results can be accurately reproduced by using exclusively $\widehat{V}_{I}$ in $(54)(\mathrm{a})$.

\section{Analysis of SUGRA Inflation}

Keeping in mind that for $K=K_{3 i}\left[K=K_{2 i}\right]$ the values $i=2$ and $8[i=3$ and 11] lead to the stabilization of $S$ during and after IGI, we proceed with the computation of the inflationary observables for the SUGRA models considered above. Since the precise choice of the index $i$ does not influence our outputs, here we do not specify henceforth the allowed $i$ values. We first present, in Section 4.1, analytic results which are in good agreement with our numerical results displayed in Section 4.2. Finally we investigate the UV behavior of the models in Section 4.3.

4.1. Analytical Results. The duration of the IGI is controlled by the slow-roll parameters, which are calculated to be

$(\widehat{\epsilon}, \widehat{\eta})$

$$
= \begin{cases}\left(\frac{2^{n}}{3 f_{\phi}^{2}}, \frac{2^{1+n / 2}\left(2^{n / 2}-c_{T} \phi^{n}\right)}{3 f_{\phi}^{2}}\right) & \text { for } K=K_{3 i} \\ \left(\frac{2^{n-2}}{f_{\phi}^{2}}, \frac{2^{n / 2}\left(2^{n / 2}-c_{T} \phi^{n}\right)}{f_{\phi}^{2}}\right) & \text { for } K=K_{2 i} .\end{cases}
$$

The end of inflation is triggered by the violation of $\widehat{\epsilon}$ condition when $\phi=\phi_{f}$ given by

$$
\begin{aligned}
\widehat{\epsilon}\left(\phi_{f}\right)=1 \Longrightarrow \\
\phi_{f} \simeq \sqrt{2} \cdot \begin{cases}\left(\frac{(1+2 / \sqrt{3})}{2 c_{T}}\right)^{1 / n} & \text { for } K=K_{3 i} \\
\left(\frac{(1+\sqrt{2})}{2 c_{T}}\right)^{1 / n} & \text { for } K=K_{2 i} .\end{cases}
\end{aligned}
$$

The violation of $\hat{\eta}$ condition occurs when $\phi=\widetilde{\phi}_{f}<\phi_{f}$ :

$$
\begin{aligned}
\widehat{\eta}\left(\widetilde{\phi}_{f}\right)=1 \Longrightarrow \\
\tilde{\phi}_{f} \simeq \sqrt{2} \cdot \begin{cases}\left(\frac{5}{6 c_{T}}\right)^{1 / n} & \text { for } K=K_{3 i} \\
\left(\frac{\sqrt{3}}{2 c_{T}}\right)^{1 / n} & \text { for } K=K_{2 i} .\end{cases}
\end{aligned}
$$

Given $\phi_{f}$, we can compute $\widehat{N}_{\star}$ via (4):

$$
\begin{aligned}
\widehat{N}_{\star}=\frac{\kappa}{2}\left(2^{1-n / 2} c_{T}\left(\phi_{\star}^{n}-\phi_{f}^{n}\right)-n \ln \frac{\phi_{\star}}{\phi_{f}}\right) \\
\text { with } \kappa= \begin{cases}\frac{3}{2} & \text { for } K=K_{3 i} \\
1 & \text { for } K=K_{2 i} .\end{cases}
\end{aligned}
$$

Ignoring the logarithmic term and taking into account that $\phi_{f} \ll \phi_{\star}$, we obtain a relation between $\phi_{\star}$ and $\widehat{N}_{\star}$ :

$$
\phi_{\star} \simeq \sqrt[n]{\frac{2^{n / 2} \widehat{N}_{\star}}{\kappa c_{T}}} .
$$


TABLE 3: Input and output parameters of the models which are compatible with (4) for $\widehat{N}_{\star}=53.2$, (6), and (7).

\begin{tabular}{|c|c|c|c|c|c|c|c|c|}
\hline \multirow{3}{*}{ Kähler potential $K$} & \multicolumn{8}{|c|}{ Parameters } \\
\hline & \multicolumn{3}{|c|}{ Input } & \multicolumn{5}{|c|}{ Output } \\
\hline & $n$ & $c_{T}$ & $\phi_{\star}$ & $\lambda\left(10^{-3}\right)$ & $\phi_{f}$ & $n_{s}$ & $\alpha_{s}\left(10^{-4}\right)$ & $r\left(10^{-3}\right)$ \\
\hline$K_{3 i}$ & 1 & 1 & 54.5 & 0.022 & 1.5 & 0.964 & -6.3 & 3.6 \\
\hline$K_{2 i}$ & 1 & 1 & 80 & 0.028 & 1.7 & 0.964 & -6.6 & 2.5 \\
\hline$K_{3 i}$ & 2 & 77 & 1 & 1.7 & 0.17 & 0.964 & -6.7 & 3.7 \\
\hline$K_{3 i}$ & 3 & 109 & 1 & 2.4 & 0.3 & 0.964 & -6.5 & 3.7 \\
\hline$K_{2 i}$ & 2 & 113 & 1 & 2 & 0.15 & 0.964 & -6.7 & 2.5 \\
\hline$K_{2 i}$ & 3 & 159 & 1 & 3 & 0.3 & 0.964 & -6.7 & 2.6 \\
\hline
\end{tabular}

Obviously, IGI, consistent with (9)(b), can be achieved if

$$
\begin{aligned}
& \phi_{\star} \leq 1 \Longrightarrow \\
& c_{T} \geq \frac{2^{n / 2} \widehat{N}_{\star}}{\kappa} .
\end{aligned}
$$

Therefore, we need relatively large $c_{T}$ 's, which increase with $n$. On the other hand, $\widehat{\phi}_{\star}$ remains trans-Planckian, since solving the first relation in (57b) with respect to $\phi$ and inserting (61a), we find

$$
\widehat{\phi}_{\star} \simeq \widehat{\phi}_{c}+\sqrt{\kappa} \ln \left(\frac{2 \widehat{N}_{\star}}{\kappa}\right) \simeq \begin{cases}5.2 & \text { for } K=K_{3 i} \\ 4.6 & \text { for } K=K_{2 i},\end{cases}
$$

where the integration constant $\widehat{\phi}_{c}=0$ and, as in the previous cases, we set $\widehat{N}_{\star} \simeq 53$. Despite this fact, our construction remains stable under possible corrections from higher order terms in $f_{K}$, since when these are expressed in terms of initial field $T$, they can be seen to be harmless for $|T| \leq 1$.

Upon substitution of (54) and (61a) into (6), we find

$$
A_{s}^{1 / 2} \simeq \begin{cases}\frac{\lambda\left(3-4 \widehat{N}_{\star}\right)^{2}}{96 \sqrt{2} \pi c_{T} \widehat{N}_{\star}} & \text { for } K=K_{3 i} \\ \frac{\lambda\left(1-2 \widehat{N}_{\star}\right)^{2}}{16 \sqrt{3} \pi c_{T} \widehat{N}_{\star}} & \text { for } K=K_{2 i} .\end{cases}
$$

Enforcing (6), we obtain a relation between $\lambda$ and $c_{T}$, which turns out to be independent of $n$. Indeed we have

$$
\lambda \simeq \begin{cases}\frac{6 \pi \sqrt{2 A_{s}} c_{T}}{\widehat{N}_{\star}} \Longrightarrow c_{T} \simeq 42969 \lambda & \text { for } K=K_{3 i} \\ \frac{4 \pi \sqrt{3 A_{s}} c_{T}}{\widehat{N}_{\star}} \Longrightarrow c_{T} \simeq 52627 \lambda & \text { for } K=K_{2 i} .\end{cases}
$$

Finally, substituting the value of $\phi_{\star}$ given in (61a) into (8), we estimate the inflationary observables. For $K=K_{3 i}$ the results are given in (31a) $-(31 \mathrm{c})$. For $K=K_{2 i}$ we obtain the relations:

$$
\begin{aligned}
& n_{s}=\frac{4 \widehat{N}_{\star}\left(\widehat{N}_{\star}-3\right)-3}{\left(1-2 \widehat{N}_{\star}\right)^{2}} \simeq 1-\frac{2}{\widehat{N}_{\star}}-\frac{3}{\widehat{N}_{\star}^{2}} \simeq 0.961 ; \\
& \alpha_{s} \simeq \frac{16 \widehat{N}_{\star}\left(3+2 \widehat{N}_{\star}\right)}{\left(2 \widehat{N}_{\star}-1\right)^{4}} \simeq-\frac{2}{\widehat{N}_{\star}^{2}}-\frac{7}{\widehat{N}_{\star}^{3}} \simeq-0.00075 ; \\
& r \simeq \frac{32}{\left(1-2 \widehat{N}_{\star}\right)^{2}} \simeq \frac{8}{\widehat{N}_{\star}^{2}}+\frac{8}{\widehat{N}_{\star}^{3}} \simeq 0.0028 .
\end{aligned}
$$

These outputs are consistent with our results in [58] for $m=n$ and $n_{11}=n_{2}=2$ (in the notation of that reference).

4.2. Numerical Results. The analytical results presented above can be verified numerically. The inflationary scenario depends on the following parameters (see (42) and (44)):

$$
n, c_{T} \text { and } \lambda
$$

Note that the stabilization of $S$ with one of $K_{32}, K_{34}, K_{23}$, and $K_{2,11}$ does not require any additional parameter. Recall that we use $T_{\mathrm{rh}}=4.1 \cdot 10^{-9}$ throughout and $\widehat{N}_{\star}$ is computed self-consistently for any $n$ via (4). Our result is $\widehat{N}_{\star} \simeq 53.2$. For given $n$, the parameters above together with $\phi_{\star}$ can be determined by imposing the observational constraints in (4) and (6). In our code we find $\phi_{\star}$ numerically, without the simplifying assumptions used for deriving (61a). Inserting it into (8), we extract the predictions of the models.

The variation of $\widehat{V}_{I}$ as a function of $\phi$ for two different values of $n$ can be easily inferred from Figure 3. In particular, we plot $\widehat{V}_{I}$ versus $\phi$ for $\phi_{\star}=1, n=2$, or $n=6$, setting $K=K_{3 i}$ in Figure 3(a) and $K=K_{2 i}$ in Figure 3(b). Imposing $\phi_{\star}=1$ for $n=2$ amounts to $\left(\lambda, c_{T}\right)=(0.0017,77)$ for $K=K_{3 i}$ and $\left(\lambda, c_{T}\right)=(0.0017,113)$ for $K=K_{2 i}$. Also, $\phi_{\star}=1$ for $n=6$ is obtained for $\left(\lambda, c_{T}\right)=(0.0068,310)$ for $K=K_{3 i}$ and $\left(\lambda, c_{T}\right)=$ $(0.0082,459)$ for $K=K_{2 i}$. In accordance with our findings in (61b), we conclude that increasing $n$ (i) requires larger $c_{T}$ 's and, therefore, lower $\widehat{V}_{I}$ 's to obtain $\phi \leq 1$; (ii) larger $\phi_{f}$ and $\langle\phi\rangle$ are obtained; see Section 4.3. Combining (59a) and (64) with (54)(a), we can conclude that $\widehat{V}_{I}\left(\phi_{f}\right)$ is independent of $c_{T}$ and to a considerable degree of $n$.

Our numerical findings for $n=1,2$, and 3 and $K=K_{3 i}$ or $K=K_{2 i}$ are presented in Table 2. In the two first rows, we present results associated with Ceccoti-like models [97], which are defined by $c_{T}=n=1$ and cannot be made consistent with the imposed $\mathbb{Z}_{n}$ symmetry or with (9). We see that, selecting $\phi_{\star} \gg 1$, we attain solutions that satisfy all the remaining constraints in Section 2.2. For the other cases, we choose a $c_{T}$ value so that $\phi_{\star}=1$. Therefore, the presented $c_{T}$ is the minimal one, in agreement with (61b).

In all cases shown in Table 2 , the model's predictions for $n_{s}, \alpha_{s}$, and $r$ are independent of the input parameters. This is due to the attractor behavior [30-32] that these models exhibit, provided that $c_{T}$ is large enough. Moreover, these 


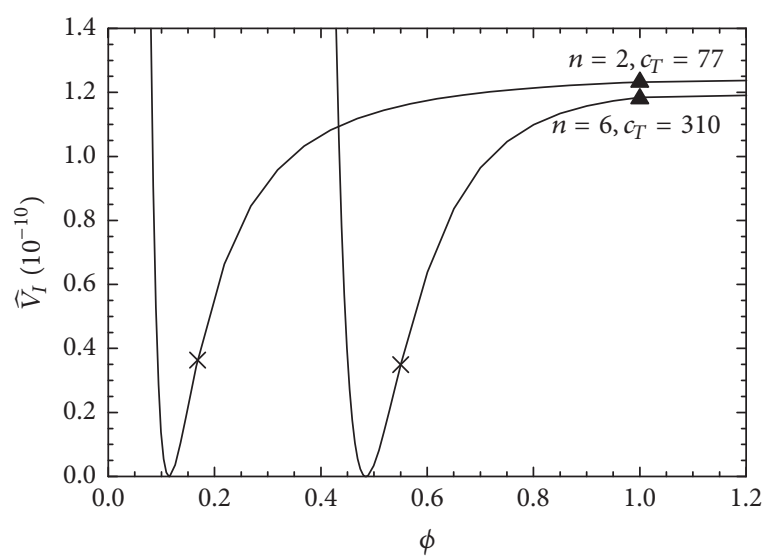

$\Delta \phi_{\star}$

$\times \phi_{f}$

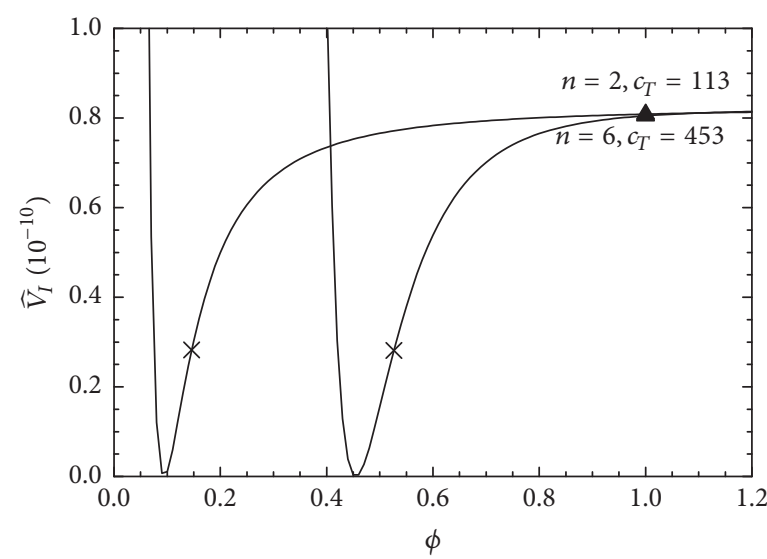

$\Delta \phi_{\star}$

$\times \phi_{f}$

(a)

(b)

FIgure 3: The inflationary potential $\widehat{V}_{I}$ as a function of $\phi$ for $\phi_{\star}=1$ and $n=2$ or $n=6$. We set (a) $K=K_{3 i}$ and (b) $K=K_{2 i}$. The values corresponding to $\phi_{\star}$ and $\phi_{f}$ are also depicted.

outputs are in good agreement with the analytical findings of (31a)-(31c) for $K=K_{3 i}$ or (65a)-(65c) for $K=K_{2 i}$. On the other hand, the presented $c_{T}, \lambda, \phi_{\star}$, and $\phi_{f}$ values depend on $n$ for every selected $K$. The resulting $n_{s} \simeq 0.964$ is close to its observationally central value; $r$ is of the order of 0.001 , and $\left|\alpha_{s}\right|$ is negligible. Although the values of $r$ lie one order of magnitude below the central value of the present combined BICEP2/Keck Array and Planck results [5], these are perfectly consistent with the $95 \%$ c.l. margin in (7). The values of $r$ for $K=K_{3 i}$ or $K=K_{2 i}$ distinguish the two cases. The difference is small, at the level of $10^{-3}$. However, it is possibly reachable by the next-generation experiment (e.g., the CMBPol experiment [98]) is expected to achieve a precision for $r$ of the order of $10^{-3}$ or even $0.5 \cdot 10^{-3}$. Finally, the renormalization scale $\Lambda$ of the ColemanWeinberg formula, found by imposing $\Delta \widehat{V}_{I}\left(\phi_{\star}\right)=0$, takes the values $7.8 \cdot 10^{-5}, 9.3 \cdot 10^{-5} 1.3 \cdot 10^{-5}$, and $2.1 \cdot 10^{-5}$ for $K_{32}$, $K_{38}, K_{23}$, and $K_{2,11}$, respectively. As a consequence, $\Lambda$ depends explicitly on the specific choice of $i$ used for $K_{3 i}$ or $K_{2 i}$.

The overall allowed parameter space of the model for $n=$ 2,3 and 6 is correspondingly

$$
77,105,310 \lesssim c_{T} \lesssim 1.6 \cdot 10^{5},
$$

$(1.7,2.4,6.8) \cdot 10^{-3} \leqslant \lambda \leqslant 3.54$

for $K=K_{3 i}$;

$$
113,159,453 \lesssim c_{T} \lesssim 1.93 \cdot 10^{5},
$$

$(2,2.9,8.2) \cdot 10^{-3} \leqslant \lambda \leqslant 3.54$

$$
\text { for } K=K_{2 i} \text {, }
$$

where the parameters are bounded from above as in (29). Letting $\lambda$ or $c_{T}$ vary within its allowed region above, we obtain the values of $n_{s}, \alpha_{s}$, and $r$ listed in Table 3 for $K=K_{3 i}$ and $K_{2 i}$ independently of $n$. Therefore, the inclusion of the variant exponent $n>2$, compared to the non-SUSY model in Section 2.4 does not affect the successful predictions of model.

4.3. UV Behavior. Following the approach described in Section 2.2, we can verify that the SUGRA realizations of IGI retain perturbative unitarity up to $m_{P}$. To this end, we analyze the small-field behavior of the theory, expanding $S$ in (1) about

$$
\langle\phi\rangle=2^{(n-2) / 2 n} c_{T}^{-1 / n},
$$

which is confined in the ranges (0.0026-0.1), (0.021-0.24), and $(0.17-0.48)$ for the margins of the parameters in (67a) and (67b).

The expansion of $\mathcal{\delta}$ is performed in terms of $\widehat{\delta \phi}$ which is found to be

$$
\widehat{\delta \phi}=\langle J\rangle \delta \phi \quad \text { with }\langle J\rangle \simeq \frac{\sqrt{\kappa} n}{\langle\phi\rangle}=2^{(2-n) / 2 n} \sqrt{\kappa} n c_{T}^{1 / n},
$$

where $\kappa$ is defined in (60). Note, in passing, that the mass of $\widehat{\delta \phi}$ at the SUSY vacuum in (41) is given by

$$
\begin{aligned}
\widehat{m}_{\delta \phi} & =\left\langle\widehat{V}_{I, \widehat{\phi} \hat{\phi}}\right\rangle^{1 / 2} \simeq \frac{\lambda}{\sqrt{2 \kappa} c_{T}} \simeq \frac{2 \sqrt{6 A_{s}} \pi}{\widehat{N}_{\star}} \\
& \simeq 1.25 \cdot 10^{-5},
\end{aligned}
$$

precisely equal to that found in (19) and (30). We observe that $\widehat{m}_{\delta \phi}$ is essentially independent of $n$ and $\kappa$, thanks to the relation between $\lambda$ and $c_{T}$ in (64). 
Expanding the second term in the r.h.s. of (33a) about $\langle\phi\rangle$ with $J$ given by the first relation in (57b), we obtain

$$
\begin{aligned}
& J^{2} \dot{\phi}^{2} \\
& =\left(1-\frac{2}{n \sqrt{\kappa}} \widehat{\delta \phi}+\frac{3}{n^{2} \kappa} \widehat{\delta \phi}^{2}-\frac{4}{n^{3}} \kappa^{-3 / 2} \widehat{\delta \phi}^{3}+\cdots\right) \\
& \quad \dot{\hat{\delta \phi}}^{2}
\end{aligned}
$$

On the other hand, $\widehat{V}_{I}$ in (54)(a) can be expanded about $\langle\phi\rangle$ as follows:

$$
\begin{aligned}
\widehat{V}_{I} & \simeq \frac{\lambda^{2} \widehat{\phi}^{2}}{4 \kappa c_{T}^{2}}\left(1-\frac{n+1}{\sqrt{\kappa} n} \widehat{\delta \phi}+(1+n) \frac{11+7 n}{12 \kappa n^{2}} \widehat{\delta \phi}^{2}\right. \\
& -\cdots) .
\end{aligned}
$$

Since the expansions above are $c_{T}$ independent, we infer that $\Lambda_{\mathrm{UV}}=1$ as in the other versions of Starobinsky-like inflation. The expansions above for $K=K_{3 i}$ and $n=2$ reduce to those in (32a) and (32b). Moreover, these are compatible with the ones presented in [31] for $K=K_{3 i}$ and those in [58] for $K=$ $K_{2 i}$ and $n_{11}=2$. Our overall conclusion is that our models do not face any problem with perturbative unitarity up to $m_{P}$.

\section{Conclusions and Perspectives}

In this review we revisited the realization of induced-gravity inflation (IGI) in both a nonsupersymmetric and supergravity (SUGRA) framework. In both cases the inflationary predictions exhibit an attractor behavior towards those of Starobinsky model. Namely, we obtained a spectral index $n_{s} \simeq(0.960-0.965)$ with negligible running $\alpha_{s}$ and a tensorto-scalar ratio $0.001 \leqslant r \leqslant 0.005$. The mass of the inflation turns out be close to $3 \cdot 10^{13} \mathrm{GeV}$. It is gratifying that IGI can be achieved for sub-Planckian values of the initial (noncanonically normalized) inflation, and the corresponding effective theories are trustable up to Planck scale, although a parameter has to take relatively high values. Moreover, the one-loop radiative corrections can be kept under control.

In the SUGRA context this type of inflation can be incarnated using two chiral superfields, $T$ and $S$, the superpotential in (42), which realizes easily the idea of induced gravity, and several (semi)logarithmic Kähler potentials $K_{3 i}$ or $K_{2 i}$; see (44). The models are pretty much constrained upon imposing two global symmetries, a continuous $R$ and a discrete $\mathbb{Z}_{n}$ symmetry, in conjunction with the requirement that the original inflation, $T$, takes sub-Planckian values. We paid special attention to the issue of $S$ stabilization during IGI and worked out its dependence on the functional form of the selected $K$ 's with respect to $|S|^{2}$. More specifically, we tested the functions $h_{i}\left(|S|^{2}\right)$, which appear in $K_{3 i}$ or $K_{2 i}$; see Table 1 . We singled out $h_{2}\left(|S|^{2}\right)$ and $h_{8}\left(|S|^{2}\right)$ for $K=K_{3 i}$ or $h_{3}\left(|S|^{2}\right)$ and $h_{11}\left(|S|^{2}\right)$ for $K=K_{2 i}$, which ensure that $S$ is heavy enough, and so well stabilized during and after inflation. This analysis provides us with new results that do not appear elsewhere in the literature. Therefore, Starobinsky inflation realized within this SUGRA set-up preserves its original predictive power, since no mixing between $|T|^{2}$ and $|S|^{2}$ is needed for consistency in the considered $K$ 's (cf. $[31,72,73]$ ).

It is worth emphasizing that the $S$-stabilization mechanisms proposed in this paper can be also employed in other models of ordinary [47-49] or kinetically modified [65-67] nonminimal chaotic (and Higgs) inflation driven by a gauge singlet $[47-49,53,54,65-67]$ or nonsinglet [50-52, 68-70] inflation, without causing any essential alteration to their predictions. The necessary modifications involve replacing the $|S|^{2}$ part of $K$ with $h_{2}\left(|S|^{2}\right)$, or $h_{8}\left(|S|^{2}\right)$ if we have a purely logarithmic Kähler potential. Otherwise, the $|S|^{2}$ part can be replaced by $h_{3}\left(|S|^{2}\right)$ or $h_{11}\left(|S|^{2}\right)$. Obviously, the last case can be employed for logarithmic or polynomial $K$ 's with regard to the inflation terms.

Let us, finally, remark that a complete inflationary scenario should specify a transition to the radiation dominated era. This transition could be facilitated in our setting $[29,62$, 63] via the process of perturbative reheating, according to which the inflation after inflation experiences an oscillatory phase about the vacuum, given by (22) for the non-SUSY case or (41) for the SUGRA case. During this phase, the inflation can safely decay, provided that it couples to light degrees of freedom in the Lagrangian of the full theory. This process is independent of the inflationary observables and the stabilization mechanism of the noninflation field. It depends only on the inflation mass and the strength of the relevant couplings. This scheme may also explain the origin of the observed baryon asymmetry through nonthermal leptogenesis, consistently with the data from the neutrino oscillations [29]. It would be nice to obtain a complete and predictable transition to the radiation dominated era. An alternative graceful exit can be achieved in the running vacuum models, as described in the fourth paper of [77-84].

\section{Conflicts of Interest}

The authors declare that there are no conflicts of interest regarding the publication of this paper.

\section{References}

[1] A. H. Guth, "Inflationary universe: a possible solution to the horizon and flatness problems," Physical Review D, vol. 23, article 347, 1981.

[2] A. D. Linde, "A new inflationary universe scenario: a possible solution of the horizon, flatness, homogeneity, isotropy and primordial monopole problems," Physics Letters B, vol. 108, no. 6, pp. 389-393, 1982.

[3] A. Albrecht and P. J. Steinhardt, "Cosmology for grand unified theories with radiatively induced symmetry breaking," Physical Review Letters, vol. 48, article 1220, 1982.

[4] P. A. R. Ade, N. Aghanim, M. Arnaud et al., "Planck 2015 results. XX. Constraints on inflation," Astronomy and Astrophysics, vol. 594, article A20, 2016.

[5] P. A. R. Ade, Z. Ahmed, R. W. Aikin et al., "Improved constraints on cosmology and foregrounds from bicep 2 and keck array cosmic microwave background data with inclusion of $95 \mathrm{GHz}$ band," Physical Review Letters, vol. 116, Article ID 031302, 2016. 
[6] K. S. Stelle, "Renormalization of higher-derivative quantum gravity," Physical Review D, vol. 16, no. 4, pp. 953-969, 1977.

[7] K. S. Stelle, "Classical gravity with higher derivatives," General Relativity and Gravitation, vol. 9, no. 4, pp. 353-371, 1978.

[8] S. V. Ketov and A. A. Starobinsky, "Embedding $R+R^{2}$ inflation in supergravity," Physical Review D, vol. 83, Article ID 063512, 2011.

[9] S. V. Ketov and N. Watanabe, "Cosmological properties of a generic Script $\mathrm{R}^{2}$-supergravity," Journal of Cosmology and Astroparticle Physics, vol. 2011, no. 3, article 11, 2011.

[10] S. V. Ketov and A. A. Starobinsky, "Inflation and nonminimal scalar-curvature coupling in gravity and supergravity," Journal of Cosmology and Astroparticle Physics, vol. 2012, no. 8, article 22, 2012.

[11] S. V. Ketov and S. Tsujikawa, "Consistency of inflation and preheating in $F(R)$ supergravity," Physical Review D, vol. 86, Article ID 023529, 2012.

[12] W. Buchmüller, V. Domcke, and K. Kamada, “The Starobinsky model from superconformal D-term inflation," Physics Letters $B$, vol. 726, no. 1-3, pp. 467-470, 2013.

[13] F. Farakos, A. Kehagias, and A. Riotto, "On the Starobinsky model of inflation from supergravity," Nuclear Physics B, vol. 876, no. 1, pp. 187-200, 2013.

[14] J. Alexandre, N. Houston, and N. E. Mavromatos, "Starobinskytype inflation in dynamical supergravity breaking scenarios," Physical Review D, vol. 89, Article ID 027703, 2014.

[15] K. Kamada and J. Yokoyama, "Topological inflation from the Starobinsky model in supergravity," Physical Review D, vol. 90, Article ID 103520, 2014.

[16] R. Blumenhagen, A. Font, M. Fuchs, D. Herschmann, and E. Plauschinn, "Towards axionic Starobinsky-like inflation in string theory," Physics Letters B, vol. 746, pp. 217-222, 2015.

[17] T. Li, Z. Li, and D. V. Nanopoulos, "Helical phase inflation via non-geometric flux compactifications: from natural to starobinsky-like inflation," Journal of High Energy Physics, vol. 2015, no. 10, article 138, 2015.

[18] S. Basilakos, N. E. Mavromatos, and J. Sola, "Starobinsky-like inflation and running vacuum in the context of supergravity," Universe, vol. 2, no. 3, article 14, 2016.

[19] J. Ellis, D. V. Nanopoulos, and K. A. Olive, "No-scale supergravity realization of the starobinsky model of inflation," Physical Review Letters, vol. 111, no. 11-13, Article ID 111301, 2013, Erratum-ibid. vol. 111, no. 12, Article ID 129902, 2013.

[20] J. Ellis, D. Nanopoulos, and K. Olive, "Starobinsky-like inflationary models as avatars of no-scale supergravity," Journal of Cosmology and Astroparticle Physics, vol. 2013, no. 10, article 9, 2013.

[21] R. Kallosh and A. Linde, "Superconformal generalizations of the Starobinsky model," Journal of Cosmology and Astroparticle Physics, vol. 2013, no. 6, article 28, 2013.

[22] D. Roest, M. Scalisi, and I. Zavala, "Kähler potentials for Planck inflation," Journal of Cosmology and Astroparticle Physics, vol. 2013, no. 11, article 7, 2013.

[23] J. Ellis, H.-J. He, and Z.-Z. Xianyu, "New Higgs inflation in a no-scale supersymmetric SU(5) GUT," Physical Review D, vol. 91, no. 2, Article ID 021302, 2015.

[24] J. Ellis, A. G. G. Marcos, N. Natsumi, V. N. Dimitri, and A. O. Keith, "Starobinsky-like inflation and neutrino masses in a no-scale SO(10) model," Journal of Cosmology and Astroparticle Physics, vol. 2016, no. 11, article 18, 2016.
[25] I. Garg and S. Mohanty, "No scale SUGRA SO(10) derived Starobinsky model of inflation," Physics Letters B, vol. 751, pp. 7-11, 2015.

[26] J. Ellis, A. G. G. Marcos, V. N. Dimitri, and A. O. Keith, "Noscale inflation," Classical and Quantum Gravity, vol. 33, no. 9, Article ID 094001, 2016.

[27] G. Chakravarty, G. Lambiase, and S. Mohanty, "Testing theories of Gravity and Supergravity with inflation and observations of the cosmic microwave background," https://arxiv.org/abs/ 1607.06325 .

[28] A. B. Lahanas and K. Tamvakis, "Inflation in no-scale supergravity," Physical Review D, vol. 91, no. 8, Article ID 085001, 2015.

[29] C. Pallis, "Linking Starobinsky-type inflation in no-scale supergravity to MSSM," Journal of Cosmology and Astroparticle Physics, vol. 2014, no. 4, article 24, 2014.

[30] G. F. Giudice and H. M. Lee, "Starobinsky-like inflation from induced gravity," Physics Letters B, vol. 733, pp. 58-62, 2014.

[31] C. Pallis, "Induced-gravity inflation in no-scale supergravity and beyond," Journal of Cosmology and Astroparticle Physics, vol. 8, article 57, 2014.

[32] R. Kallosh, "More on universal superconformal attractors," Physical Review D, vol. 89, Article ID 087703, 2014.

[33] F. S. Accetta, D. J. Zoller, and M. S. Turner, "Induced-gravity inflation," Physical Review D, vol. 31, no. 12, pp. 3046-3051, 1985.

[34] D. S. Salopek, J. R. Bond, and J. M. Bardeen, "Designing density fluctuation spectra in inflation," Physical Review D, vol. 40, no. 6, pp. 1753-1788, 1989.

[35] R. Fakir and W. G. Unruh, "Induced-gravity inflation," Physical Review D, vol. 41, no. 6, pp. 1792-1795, 1990.

[36] J. L. Cervantes-Cota and H. Dehnen, "Induced gravity inflation in the SU(5) GUT," Physical Review D, vol. 51, no. 2, article 395, 1995.

[37] N. Kaloper, L. Sorbo, and J. Yokoyama, "Inflation at the GUT scale in a Higgsless universe," Physical Review D, vol. 78, no. 4, Article ID 043527, 2008.

[38] A. Cerioni, F. Finelli, A. Tronconi, and G. Venturi, "Inflation and reheating in spontaneously generated gravity," Physical Review D, vol. 81, no. 12, Article ID 123505, 2010.

[39] K. Kannike, G. Hütsi, L. Pizza et al., "Dynamically induced Planck scale and inflatio," Journal of High Energy Physics, vol. 2015, no. 5, article 65, 2015.

[40] M. B. Einhorn and D. R. T. Jones, "Induced gravity I: real scalar field," Journal of High Energy Physics, vol. 2016, no. 1, article 19, 2016.

[41] A. Zee, "Broken-symmetric theory of gravity," Physical Review Letters, vol. 42, article 417, 1979.

[42] H. Terazawa, "Cosmological origin of mass scales," Physics Letters B, vol. 101, no. 1-2, pp. 43-47, 1981.

[43] J. L. Cervantes-Cota and H. Dehnen, "Induced gravity inflation in the standard model of particle physics," Nuclear Physics B, vol. 442, no. 1-2, pp. 391-409, 1995.

[44] F. L. Bezrukov and M. Shaposhnikov, "The Standard Model Higgs boson as the inflaton," Physics Letters B, vol. 659, no. 3, pp. 703-706, 2008.

[45] C. Pallis, "Non-minimally gravity-coupled inflationary models," Physics Letters B, vol. 692, no. 5, pp. 287-296, 2010.

[46] R. Kallosh, A. Linde, and D. Roest, "Universal attractor for inflation at strong coupling," Physical Review Letters, vol. 112, Article ID 011303, 2014. 
[47] M. B. Einhorn and D. R. T. Jones, "Inflation with non-minimal gravitational couplings in supergravity," Journal of High Energy Physics, vol. 2010, no. 3, article 26, 2010.

[48] S. Ferrara, R. Kallosh, A. Linde, A. Marrani, and A. Van Proeyen, "Superconformal symmetry, NMSSM, and inflation," Physical Review D, vol. 83, no. 2, Article ID 025008, 2011.

[49] C. Pallis and N. Toumbas, "Non-minimal sneutrino inflation, Peccei-Quinn phase transition and non-thermal leptogenesis," Journal of Cosmology and Astroparticle Physics, vol. 2011, no. 2, article 19, 2011.

[50] M. Arai, S. Kawai, and N. Okada, "Higgs inflation in minimal supersymmetric SU(5) grand unified theory," Physical Review D, vol. 84, no. 12, Article ID 123515, 2011.

[51] C. Pallis and N. Toumbas, "Non-minimal Higgs inflation and non-thermal leptogenesis in a supersymmetric Pati-Salam model," Journal of Cosmology and Astroparticle Physics, vol. 2011, no. 12, article 2, 2011.

[52] M. B. Einhorn and D. R. T. Jones, "GUT scalar potentials for Higgs inflation," Journal of Cosmology and Astroparticle Physics, vol. 2012, no. 11, article 49, 2012.

[53] C. Pallis and Q. Shafi, "Nonminimal chaotic inflation, PecceiQuinn phase transition, and nonthermal leptogenesis," Physical Review D, vol. 86, no. 2, Article ID 023523, 2012.

[54] C. Pallis and Q. Shafi, "Gravity waves from non-minimal quadratic inflation," Journal of Cosmology and Astroparticle Physics, vol. 2015, no. 3, article 23, 2015.

[55] J. L. F. Barbon and J. R. Espinosa, "On the naturalness of Higgs inflation,” Physical Review D, vol. 79, Article ID 081302, 2009.

[56] C. P. Burgess, H. M. Lee, and M. Trott, "On Higgs inflation and naturalness," Journal of High Energy Physics, vol. 2010, no. 7, article 7, 2010.

[57] A. Kehagias, A. M. Dizgah, and A. Riotto, "Remarks on the Starobinsky model of inflation and its descendants," Physical Review D, vol. 89, no. 4, Article ID 043527, 2014.

[58] C. Pallis and N. Toumbas, "Starobinsky-type inflation with products of Kähler manifolds," Journal of Cosmology and Astroparticle Physics, vol. 2016, no. 5, article 15, 2016.

[59] E. Cremmer, S. Ferrara, C. Kounnas, and D. V. Nanopoulos, "Naturally vanishing cosmological constant in $N=1$ supergravity," Physics Letters. B, vol. 133, no. 1-2, pp. 61-66, 1983.

[60] J. R. Ellis, A. B. Lahanas, D. V. Nanopoulos, and K. Tamvakis, "No-scale supersymmetric standard model," Physics Letters B, vol. 134, no. 6, pp. 429-435, 1984.

[61] A. B. Lahanas and D. V. Nanopoulos, "The road to no scale supergravity," Physics Reports, vol. 145, no. 1-2, pp. 1-139, 1987.

[62] T. Terada, Y. Watanabe, Y. Yamada, and J. Yokoyama, "Reheating processes after Starobinsky inflation in old-minimal supergravity," Journal of High Energy Physics, vol. 2015, no. 2, article 105, 2015.

[63] J. Ellis, M. Garcia, D. Nanopoulos, and K. Olive, "Phenomenological aspects of no-scale inflation models," Journal of Cosmology and Astroparticle Physics, vol. 2015, no. 10, article 3, 2015.

[64] H. M. Lee, "Chaotic inflation in Jordan frame supergravity," Journal of Cosmology and Astroparticle Physics, vol. 2010, no. 8, article 3, 2010.

[65] C. Pallis, "Kinetically modified nonminimal chaotic inflation," Physical Review D, vol. 91, no. 12, Article ID 123508, 2015.

[66] C. Pallis, "Observable gravitational waves from kinetically modified non-minimal inflation," PoS PLANCK, vol. 2015, article 95, 2015.
[67] C. Pallis, "Kinetically modified non-minimal inflation with exponential frame function," https://arxiv.org/abs/1611.07010.

[68] G. Lazarides and C. Pallis, "Shift symmetry and Higgs inflation in supergravity with observable gravitational waves," Journal of High Energy Physics, vol. 2015, no. 11, article 114, 2015.

[69] C. Pallis, "Kinetically modified nonminimal Higgs inflation in supergravity," Physical Review D, vol. 92, no. 12, Article ID 121305, 2015.

[70] C. Pallis, "Variants of kinetically modified non-minimal Higgs inflation in supergravity," Journal of Cosmology and Astroparticle Physics, vol. 2016, no. 10, article 37, 2016.

[71] C. Kounnas, D. Lüst, and N. Toumbas, "R2 inflation from scale invariant supergravity and anomaly free superstrings with fluxes," Fortschritte der Physik, vol. 63, no. 1, pp. 12-35, 2015.

[72] C. Pallis, "Reconciling induced-gravity inflation in supergravity with the Planck 2013 \& BICEP2 results," Journal of Cosmology and Astroparticle Physics, vol. 2014, no. 10, article 58, 2014.

[73] C. Pallis, "Induced-gravity inflation in supergravity confronted with planck 2015 \& BICEP2/keck array," POS CORFU, vol. 2014, article 156, 2015.

[74] C. Pallis, "Models of non-minimal chaotic inflation in supergravity," PoS CORFU, vol. 2013, article 61, 2013.

[75] I. Antoniadis, E. Dudas, S. Ferrara, and A. Sagnotti, "The Volkov-Akulov-Starobinsky supergravity," Physics Letters B, vol. 733, pp. 32-35, 2014.

[76] A. A. Starobinsky, "A new type of isotropic cosmological models without singularity," Physics Letters B, vol. 91, no. 1, pp. 99-102, 1980.

[77] J. A. S. Lima, S. Basilakos, and J. Solà, "Expansion history with decaying vacuum: a complete cosmological scenario," Monthly Notices of the Royal Astronomical Society, vol. 431, no. 1, pp. 923929, 2013.

[78] J. A. Lima, S. Basilakos, and J. Solà, "Nonsingular decaying vacuum cosmology and entropy production," General Relativity and Gravitation, vol. 47, no. 4, article 40, 2015.

[79] J. Solà and A. Gómez-Valent, "The $\bar{\Lambda}$ cosmology: from inflation to dark energy through running $\Lambda$," International Journal of Modern Physics. D. Gravitation, Astrophysics, Cosmology, vol. 24, no. 4, Article ID 1541003, 37 pages, 2015.

[80] J. A. S. Lima, S. Basilakos, and J. Solà, "Thermodynamical aspects of running vacuum models," The European Physical Journal C, vol. 76, no. 4, article 228, 2016.

[81] J. Solà, A. Gómez-Valent, and J. de Cruz Pérez, "First evidence of running cosmic vacuum: challenging the concordance model," The Astrophysical Journal, vol. 836, no. 1, article 43, 2017.

[82] J. Solà, A. Gómez-Valent, and J. de Cruz Pérez, "Hints of dynamical vacuum energy in the expanding universe," The Astrophysical Journal Letters, vol. 811, p. L14, 2015.

[83] J. Solà, J. de Cruz Pérez, A. Gómez-Valent, and R. C. Nunes, "Dynamical vacuum against a rigid cosmological constant," https://arxiv.org/abs/1606.00450.

[84] J. Solà, "Dark energy: a quantum fossil from the inflationary Universe?" Journal of Physics A: Mathematical and Theoretical, vol. 41, no. 16, Article ID 164066, 2008.

[85] D. H. Lyth and A. Riotto, "Particle physics models of inflation and the cosmological density perturbation," Physics Reports. A Review Section of Physics Letters, vol. 314, no. 1-2, pp. 1-146, 1999.

[86] G. Lazarides, "Basics of inflationary cosmology," Journal of Physics: Conference Series, vol. 53, p. 528, 2006. 
[87] A. Mazumdar and J. Rocher, "Particle physics models of inflation and curvaton scenarios," Physics Reports. A Review Section of Physics Letters, vol. 497, no. 4-5, pp. 85-215, 2011.

[88] J. Martin, C. Ringeval, and V. Vennin, "Encyclopædia inflationaris," Physics of the Dark Universe, vol. 5-6, pp. 75-235, 2014.

[89] M. S. Turner, "Coherent scalar-field oscillations in an expanding universe," Physical Review. D. Particles and Fields. Third Series, vol. 28, no. 6, pp. 1243-1247, 1983.

[90] P. A. R. Ade, N. Aghanim, M. Arnaud et al., "Planck 2015 results. XIII. Cosmological parameters," Astronomy and Astrophysics, vol. 594, article A13, p. 63, 2016.

[91] S. Renaux-Petel and K. Turzynski, "Geometrical destabilization of inflation," Physical Review Letters, vol. 117, no. 14, Article ID 141301, 2016.

[92] J. Ellis, M. A. G. García, D. V. Nanopoulos, and K. A. Olive, "Two-field analysis of no-scale supergravity inflation," Journal of Cosmology and Astroparticle Physics, vol. 2015, no. 1, article 10, 2015.

[93] C. van de Bruck and L. E. Paduraru, "Simplest extension of Starobinsky inflation," Physical Review D, vol. 92, no. 8, Article ID 083513, 2015.

[94] S. Kaneda and S. V. Ketov, "Starobinsky-like two-field inflation," The European Physical Journal C, vol. 76, no. 1, p. 26, 2016.

[95] G. Chakravarty, S. Das, G. Lambiase, and S. Mohanty, "Dilaton assisted two-field inflation from no-scale supergravity," Physical Review D, vol. 94, no. 2, Article ID 023521, 2016.

[96] S. R. Coleman and E. J. Weinberg, "Radiative corrections as the origin of spontaneous symmetry breaking," Physical Review D, vol. 7, no. 6, pp. 1888-1910, 1973.

[97] S. Cecotti, "Higher derivative supergravity is equivalent to standard supergravity coupled to matter," Physics Letters. B. Particle Physics, Nuclear Physics and Cosmology, vol. 190, no. 1-2, pp. 86-92, 1987.

[98] D. Baumann, M. G. Jackson, P. Adshead, A. Amblard, and A. Ashoorioon, "Probing inflation with CMB polarization," AIP Conference Proceedings, vol. 1141, article 10, 2009. 

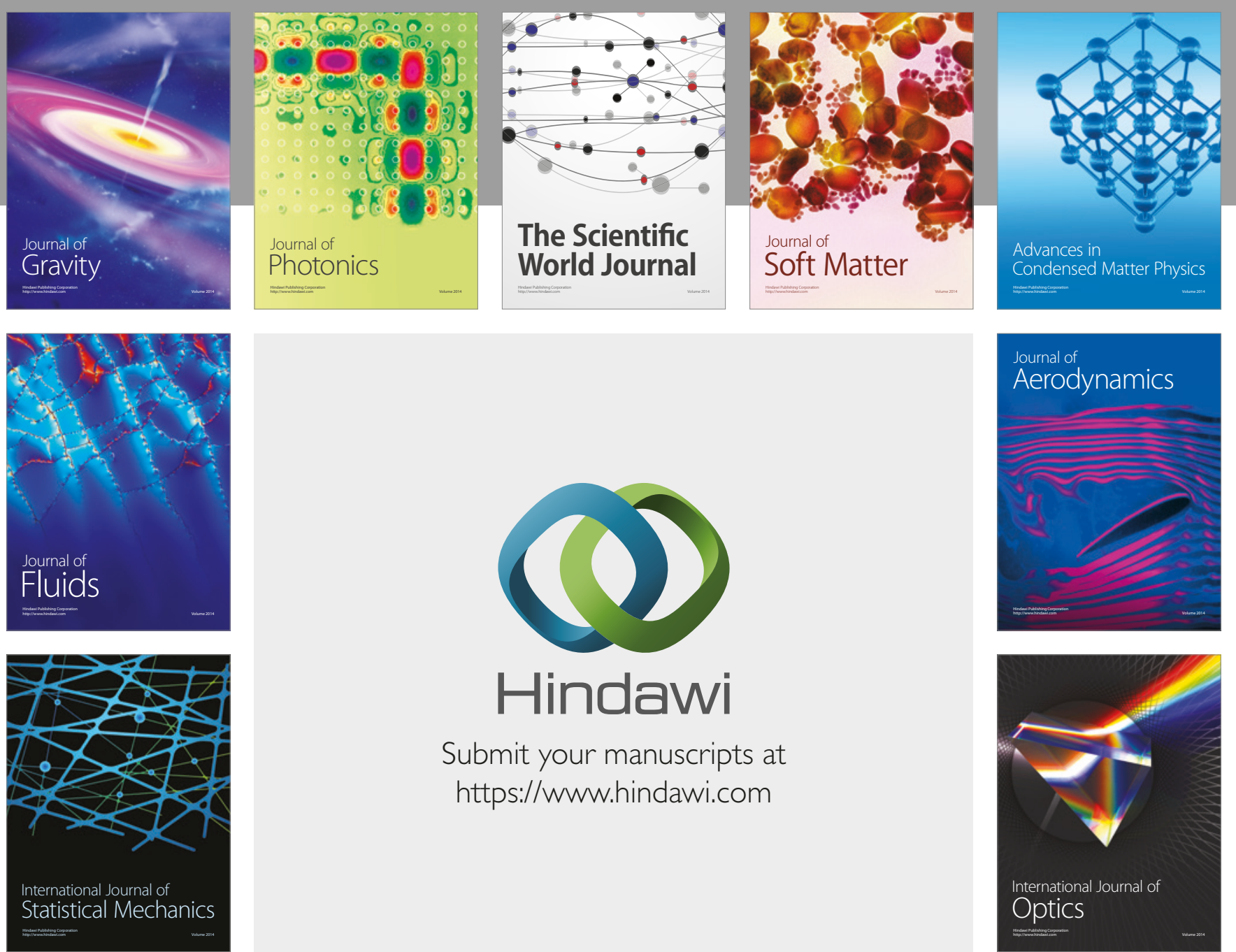

Submit your manuscripts at

https://www.hindawi.com
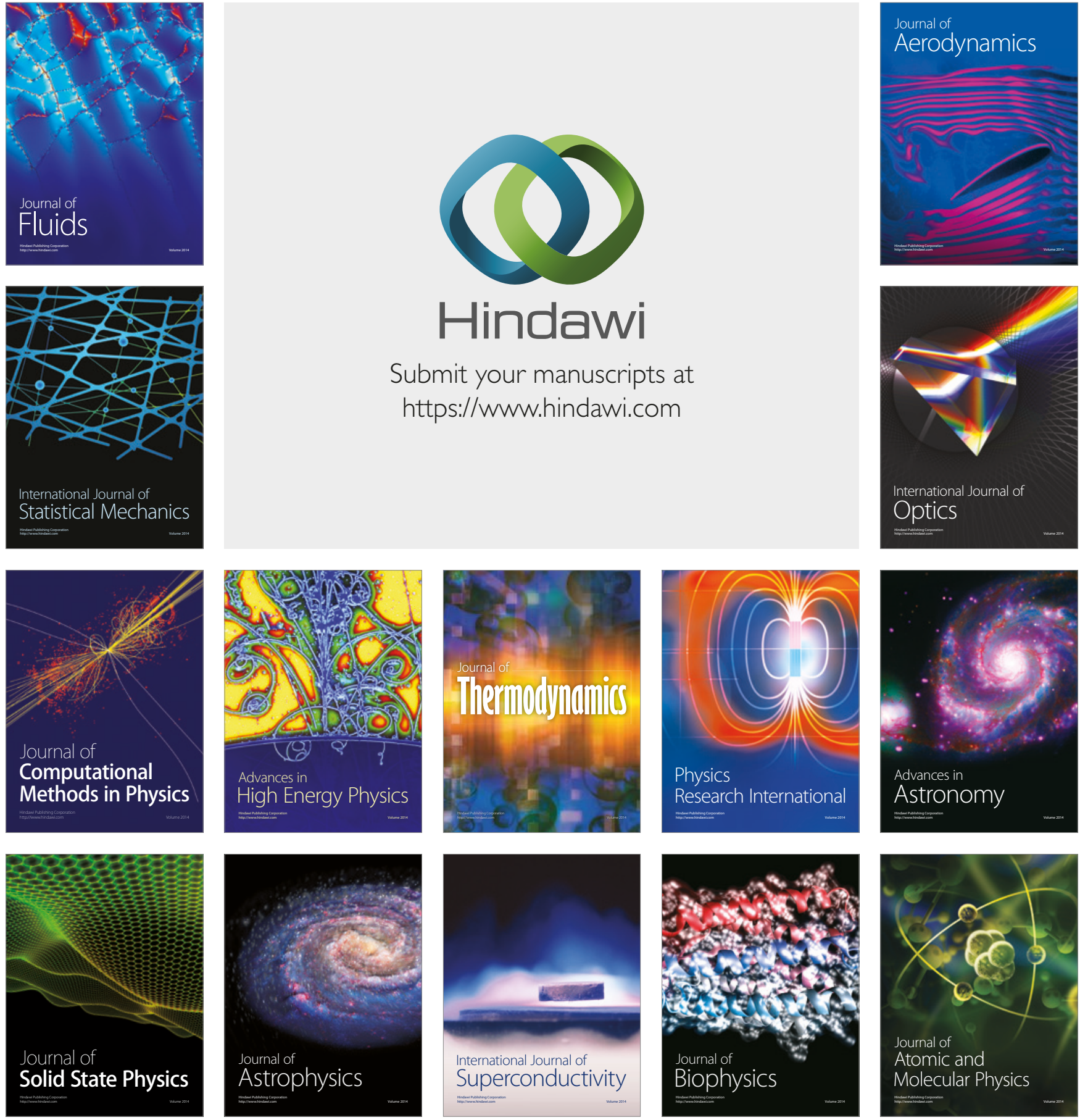